\title{
Data-Driven Generalized Integer Aperture Bootstrapping for Real-Time High Integrity Applications
}

\author{
G. Nathan Green, The University of Texas at Austin \\ Martin King, NAVAIR \\ Dr. Todd Humphreys, The University of Texas at Austin
}

\begin{abstract}
A new method is developed for integer ambiguity resolution in carrier-phase differential GPS (CDGPS) positioning. The method is novel in that it is (1) data-driven, (2) generalized to include partial ambiguity resolution, and (3) amenable to a full characterization of the prior and posterior distributions of the three-dimensional baseline vector that results from CDGPS. The technique is termed generalized integer aperture bootstrapping (GIAB). GIAB improves the availability of integer ambiguity resolution for high-integrity, safety-critical systems. Current high-integrity CDGPS algorithms, such as EPIC and GERAFS, evaluate the prior risk of position domain biases due to incorrect integer ambiguity resolution without further validation of the chosen solution. This model-driven approach introduces conservatism which tends to reduce solution availability. Common data-driven ambiguity validation methods, such as the ratio test, control the risk of incorrect ambiguity resolution by shrinking an integer aperture (IA), or acceptance region. The incorrect fixing risk of current IA methods is determined by functional approximations that are inappropriate for use in safety-of-life applications. Moreover, generalized IA (GIA) methods incorrectly assume that the baseline resulting from partial ambiguity resolution is zero mean. Each of these limitations is addressed by GIAB, and the claimed improvements are validated by Monte Carlo simulation. The performance of GIAB is then optimized by tuning the integer aperture size to maximize the prior probability of full ambiguity resolution. GIAB is shown to provide higher availability than EPIC for the same integrity requirements.
\end{abstract}

Keywords-Generalized integer aperture, bootstrap, CDGPS, integrity, availability, partial ambiguity resolution, LAMBDA, data-driven, EPIC, GERAFS

\section{INTRODUCTION}

The required navigation performance for CDGPS systems has become more demanding with each new application. Performance is assessed in terms of integrity, accuracy, continuity, and availability. Integrity is specified in terms of integrity risk $(I R)$, the probability that the solution error exceeds an alert limit $(A L)$ without warning. Accuracy can be specified in terms of quantiles of interest, such as $95 \%$ accuracy, which refers to the error volume within which $95 \%$ of solutions fall. Continuity risk $(C R)$ is the probability that the solution will become unavailable during a critical exposure interval given that it was available at the beginning of that interval. Availability is the percentage of time that the solution satisfies its required integrity, accuracy, and continuity requirements.

The ground-based augmentation system (GBAS), originally specified over a decade ago as a landing aid for large runways on land, specified $A L \mathrm{~s}$ of $10 \mathrm{~m}$ with $I R$ on the order of
$10^{-7}$ per approach. This leads to a relatively loose $95 \%$ accuracy requirement of $2 \mathrm{~m}$ for a zero-mean-error Gaussiandistributed solution, which can be met by a float CDGPS solution. More recent navigation system applications, such as landing aboard an aircraft carrier and a recent demonstration of autonomous aerial refueling, have meter-level $A L \mathrm{~s}, I R$ on the order of $10^{-6}$, and decimeter-level accuracy requirements. Such a stringent performance specification can only be met by CDGPS positioning when the integer ambiguities are resolved.

The next generation of CDGPS use cases includes fully autonomous landing and refueling of large, unmanned aerial vehicles (UAVs) in operational contexts, which will demand $C R$ lower than all previous applications, $I R$ on the order of $10^{-7}$, and decimeter-level accuracy requirements. For these demanding new applications to achieve the required high availability, low $I R$, and excellent accuracy, it will be essential to precisely control the risk of incorrect ambiguity resolution while reducing and bounding any residual biases in the relative navigation solution.

State-of-the-art methods in high-integrity CDGPS enforce the $I R$ constraint in the position domain by accounting for relative position biases induced by incorrect fixing. Two such methods are the Geometry Extra Redundant Almost Fixed Solutions (GERAFS) [1] and the Enforced Position-domain Integrity-risk of Cycle resolution (EPIC) [2], [3] algorithms. Both of these rely exclusively on a priori error models to determine, before the measurements are processed, whether a fixed solution or a float backup solution will be used. This approach is termed model-driven because the solution selection logic is entirely dependent on the prior error model. Because the EPIC and GERAFS algorithms attempt to bound $I R$ using the $a$ priori distribution, they are inherently conservative. They must evaluate the position domain biases induced by a large number of potential incorrect fixes. The biases lead to large potential errors that must be protected against without knowledge of the observed carrier phase measurements.

In contrast to the model-driven approach, data-driven methods decide a posteriori whether to accept the fixed or float solution. Conditioning the selection on the observed measurements reduces the risk of incorrect ambiguity resolution. A subset of data-driven methods is called integer aperture (IA) estimation. In IA methods, the integer ambiguity is estimated using either bootstrapping [4] or integer least squares (ILS) [5], after which a test statistic is computed using the ambi- 
guity residual, i.e., the difference between the float and fixed ambiguities. This test statistic is compared to a threshold to decide between the fixed and float solution.

Perhaps the simplest IA method is IA bootstrapping (IAB), which resolves the integer ambiguities via integer bootstrapping and then tests the fixed solution by applying bootstrapping to a scaled-up version of the ambiguity residual [6]. If the test returns the zero vector, then the fixed solution is selected; otherwise the float solution is selected. IAB is sub-optimal in the sense that bootstrapping does not always find the maximum likelihood integer ambiguity (as opposed to ILS, which is guaranteed to do so). It is also sub-optimal in the sense that it does not maximize the probability of successfully fixing the ambiguities for a given probability of incorrectly fixing them. But it has the advantage that all of these probabilities have analytically computable values, which allows the decision threshold to be set analytically and, more generally, enables the strict performance requirements that safety-of-life applications demand to be provably satisfied.

The remaining IA methods discussed in this introduction use ILS to solve for the integer ambiguity, which is optimal in the maximum likelihood sense for Gaussian measurement noise. Ellipsoidal IA uses the covariance weighted norm of the ILS ambiguity residual as a test statistic [7]. The simplicity of this statistic allows the decision threshold to be set analytically, as with IA bootstrapping, but the probability of successfully fixing the ambiguities is also sub-optimal. While ellipsoidal IA can have a higher probability of success than IAB for models with a few ambiguities of approximately equal conditional variance, IAB tends to provide a higher probability of success for models in which the conditional variances of the ambiguities differ by more than about $10 \%$, which is smaller than typical differences in realistic measurement models.

There are several other ILS-based IA methods that employ test statistics that are a function of the ambiguity residuals of the ILS fix and of one or more alternate fixes with the next lowest cost. These methods include the ratio test [8], the difference test [9], and an optimal test [10]. Unlike IA bootstrapping and ellipsoidal IA, none of these methods' test statistics has an analytical probability distribution or decision threshold [11]. In practice, decision thresholds are set based on one of a few ad hoc methods. The crudest method is to apply a fixed threshold for all measurement models, but this prevents precise control of the risk of incorrect fix for time-varying measurement models and satellite geometry. Methods that set thresholds to control the risk of incorrect fix based on the (time-varying) measurement model are needed to control IR. Such methods include threshold determination via Monte Carlo simulation, lookup tables [8], and functional approximations [12], [13].

The Monte Carlo simulations, lookup tables, and functional approximations that are used to set aperture thresholds in the ILS-based IA methods are inapt for safety-of-life systems. The thresholds that result from these methods cannot be analytically proven to bound the risk of incorrect fix for particular models. At best, they incorporate sufficient conservatism to protect the solution at the expense of decreased availability. Also, Monte Carlo simulations particular to the measurement model in use are not feasible for a real-time solution.

The optimal IA algorithm uses as its test statistic the $a$ posteriori probability of correct fix. Contrary to intuition, the threshold for a particular probability of incorrect ambiguity resolution for this statistic is not analytically computable [14]. Also, the optimal IA estimator involves an infinite sum over all possible integer ambiguities. The search can be truncated once a sufficiently large number of integer fixes has been evaluated, but the number required depends on strength of the model and on the required $I R$ required. The search often extends to several hundred candidate fixes in realistic simulations to satisfy the most demanding integrity requirements, which becomes impractical for real-time applications.

When IA estimators are extended from resolution of the full set of integer ambiguities to subsets of the full set, they are called Generalized Integer Aperture (GIA) estimators [15]. If the full set of ambiguities is unable to be fixed, these algorithms evaluate successively smaller subsets until either a satisfactory fix is found or the float solution is applied as a last resort. Partial ambiguity resolution offers gradual degradation of performance for weak models. To the best of the authors' knowledge, no previous work has accurately described the distribution of the data-driven, partially fixed baseline.

In summary, to meet the increasingly stringent performance requirements of safety-of-life applications there is a need for a data-driven ambiguity resolution and validation method whose decision threshold for choosing between a fixed and float solution can be set analytically. The method must be generalized to accommodate partial ambiguity resolution, and must correctly characterize the distribution of the data-driven, partially-fixed baseline. Extant methods in the high-integrity CDGPS literature do not satisfy this need.

This paper offers three contributions to address this need. First, IAB is extended to encompass partial ambiguity resolution. The extended technique will be called Generalized Integer Aperture Bootstrapping (GIAB). Second, an analytical characterization of the a priori and a posteriori distributions of the GIAB baseline is developed and validated. In passing, existing GIA methods are shown to incorrectly neglect solution biases. Third, a method for setting the integer aperture size and shape is developed that maximizes solution availability subject to a constraint on the risk of incorrect ambiguity resolution. These contributions are validated with a set of Monte Carlo simulations, and algorithm performance is compared to the state-of-the-art EPIC algorithm to demonstrate the advantages of the new method. EPIC is the focus of comparison because it provides higher availability than GERAFS and is appropriate for safety-of-life applications. However, since EPIC is modeldriven, GIAB is shown to provide higher availability for the same $I R$ and $A L$.

\section{Generalized Integer Aperture Bootstrapping}

\section{A. Integer Bootstrapping Overview}

The most important results of integer bootstrapping (IB) are reproduced here from [4] with a few amplifications for ease of reference and notational consistency. IB operates by sequentially rounding one integer ambiguity and conditioning the remaining float ambiguities on the assumption that 
the preceding fixes were all correct. IB should always be used in conjunction with the decorrelating Z-transform of the LAMBDA method to maximize the probability of success [16]. The float baseline, the decorrelated float ambiguities, and the joint covariance matrix of the two vectors are denoted by

$$
\begin{gathered}
\hat{\boldsymbol{x}}=\left[\begin{array}{c}
\hat{\boldsymbol{b}} \\
\hat{\boldsymbol{z}}
\end{array}\right] \\
\hat{\boldsymbol{b}} \in \mathbb{R}^{3}: \text { float estimate of the baseline vector } \\
\hat{z} \in \mathbb{R}^{n_{\mathrm{z}}}: \text { decorrelated float ambiguity vector } \\
\mathbf{Q}_{\hat{\boldsymbol{x}}}=\left[\begin{array}{cc}
\mathbf{Q}_{\hat{\boldsymbol{b}}} & \mathbf{Q}_{\hat{\boldsymbol{b}} \hat{z}} \\
\mathbf{Q}_{\hat{z} \hat{b}} & \mathbf{Q}_{\hat{z}}
\end{array}\right] \\
\mathbf{Q}_{\hat{\boldsymbol{b}}}: \text { Covariance of the float baseline estimate } \\
\mathbf{Q}_{\hat{\boldsymbol{b}} \hat{\boldsymbol{z}}}=\mathbf{Q}_{\hat{z} \hat{\boldsymbol{b}}}^{T}: \text { Covariance of float baseline and ambiguity } \\
\mathbf{Q}_{\hat{\boldsymbol{z}}}: \text { Covariance of the float ambiguity estimate }
\end{gathered}
$$

The covariance of the float ambiguity can be decomposed as $\mathbf{Q}_{\hat{\boldsymbol{z}}}=L D L^{T}$, where $L$ is a unit lower triangular matrix and $D$ is a diagonal matrix. Let $l_{i j}$ denote the $i j^{t h}$ element of $L$ and $d_{i}$ the $i^{t h}$ element of the diagonal of $D$.

The float integer ambiguity is modeled as the true ambiguity plus zero-mean Gaussian noise: $\hat{\boldsymbol{z}}=\boldsymbol{z}+\boldsymbol{\epsilon}, \boldsymbol{\epsilon} \sim \mathcal{N}\left(0, \mathbf{Q}_{\hat{z}}\right)$. Multiplication by $L^{-1}$ transforms $\epsilon$ into a vector whose elements are mutually uncorrelated: $\boldsymbol{\epsilon}_{\mathrm{c}}=L^{-1} \boldsymbol{\epsilon}, \boldsymbol{\epsilon}_{\mathrm{c}} \sim \mathcal{N}(0, D)$. Since $L$ and $L^{-1}$ are unit lower triangular matrices, the $i^{\text {th }}$ component of $\boldsymbol{\epsilon}$, denoted $\epsilon_{i}$, and its variance, $\operatorname{var}\left(\epsilon_{i}\right)$, can be computed from the first $i$ components of $\epsilon_{\mathrm{c}}$ :

$$
\epsilon_{i}=\epsilon_{\mathrm{c}, i}+\sum_{j=1}^{i-1} l_{i j} \epsilon_{\mathrm{c}, j}
$$

and

$$
\begin{aligned}
\operatorname{var}\left(\epsilon_{i}\right) & =\operatorname{var}\left(\epsilon_{\mathrm{c}, i}\right)+\sum_{j=1}^{i-1} l_{i j}^{2} \operatorname{var}\left(\epsilon_{\mathrm{c}, j}\right) \\
& =d_{i}+\sum_{j=1}^{i-1} l_{i j}^{2} d_{j}
\end{aligned}
$$

This construction gives rise to the interpretation of $L$ as the sequential conditional correlations among the ambiguities, of $D$ as the sequential conditional variances of the ambiguities, and of $\epsilon_{\mathrm{c}}$ as the conditional ambiguity residuals:

$$
d_{i}=\sigma_{i \mid I}^{2}
$$

and

$$
l_{i j}=\left\{\begin{array}{lr}
\sigma_{i, j \mid J}^{2} \sigma_{j \mid J}^{-2} & j \leq i \\
0 & \text { otherwise }
\end{array}\right.
$$

where $\sigma_{i \mid I}^{2}$ is the variance of $\hat{z}_{i}$ conditioned on the previous $i-1$ ambiguities, and $\sigma_{i, j \mid J}^{2}$ is the covariance of $\hat{z}_{i \mid J}$ and $\hat{z}_{j \mid J}$ conditioned on the previous $j-1$ ambiguities.

The integer bootstrap algorithm proceeds by alternating rounding and conditioning steps. First, the ambiguity with the lowest variance is rounded to the nearest integer. Next, the first ambiguity residual is computed and used to condition the remaining float ambiguities. These steps are repeated for the remaining ambiguities one by one: The ambiguity with the lowest conditional variance is rounded, the conditioned residual is computed, and the rest of the ambiguities are further conditioned. The use of the ambiguity decorrelation Z-transform and the $L D L^{T}$ decomposition ensures that the transformed integers are ordered such that fixing from first to last is the correct order.

Let the integer bootstrapping process be represented by the functional map

$$
\breve{z}=\operatorname{IB}(\hat{z}): \mathbb{R}^{n} \mapsto \mathbb{Z}^{n}
$$

There are two equivalent ways to perform this operation, depending on the decomposition of $\mathbf{Q}_{\hat{z}}$ used. The pseudocode for both is given below because both provide insights that are useful later in this paper. The operation $\lfloor\cdot 7$ is the nearest integer rounding operation. In the second option, $\ell_{i j}$ is the $i j^{t h}$ element of $L^{-1}$. The notation $\breve{\boldsymbol{\epsilon}}=\hat{\boldsymbol{z}}-\breve{z}$ denotes an estimate of the ambiguity residual, assuming that the fixed integers are correct. Similarly, $\breve{\boldsymbol{\epsilon}}_{\mathrm{c}}=\hat{\boldsymbol{z}}_{\mathrm{c}}-\breve{\boldsymbol{z}}$ denotes an estimate of the sequentially-conditioned ambiguity residual, assuming that the fixed integers are correct.

$$
\begin{array}{l|l}
\begin{array}{l|l}
\text { Option 1: } \\
\text { for } i=1: n_{\mathrm{z}}
\end{array} & \begin{array}{l}
\text { Option 2: } \\
\text { for } i=1: n_{\mathrm{z}}
\end{array} \\
\hat{z}_{\mathrm{c}, i}=\hat{z}_{i}-\sum_{j=1}^{i-1} l_{i j} \breve{\epsilon}_{\mathrm{c}, j} & \hat{z}_{\mathrm{c}, i}=\hat{z}_{i}+\sum_{j=1}^{i-1} \ell_{i j} \breve{\epsilon}_{j} \\
\breve{z}_{i}=\left\lfloor\hat{z}_{\mathrm{c}, i}\right\rceil & \breve{z}_{i}=\left\lfloor\hat{z}_{\mathrm{c}, i}\right\rceil \\
\begin{array}{l}
\breve{\epsilon}_{\mathrm{c}, i}=\hat{z}_{\mathrm{c}, i}-\breve{z}_{i} \\
\text { end }
\end{array} & \begin{array}{c}
\breve{\epsilon}_{i}=\hat{z}_{i}-\breve{z}_{i} \\
\text { end }
\end{array}
\end{array}
$$

In the first option, the conditional ambiguity residual, $\breve{\epsilon}_{\mathrm{c}}$, is computed. In the second option, the unconditional ambiguity residual, $\breve{\boldsymbol{\epsilon}}$, is computed. Once the final fixed solution is computed, the float baseline is conditioned on the chosen integers as follows:

$$
\begin{aligned}
\breve{\boldsymbol{b}} & =\hat{\boldsymbol{b}}-\mathbf{Q}_{\hat{\boldsymbol{b}} \hat{\boldsymbol{z}}} \mathbf{Q}_{\hat{\boldsymbol{z}}}^{-1} \breve{\boldsymbol{\epsilon}} \\
& =\hat{\boldsymbol{b}}-\mathbf{Q}_{\hat{\boldsymbol{b}} \hat{\boldsymbol{z}}} L^{-T} D^{-1} \breve{\boldsymbol{\epsilon}}_{\mathrm{c}} \\
\mathbf{Q}_{\breve{\boldsymbol{b}}} & =\mathbf{Q}_{\hat{\boldsymbol{b}}}-\mathbf{Q}_{\hat{\boldsymbol{b}} \hat{\boldsymbol{z}}} \mathbf{Q}_{\hat{\boldsymbol{z}}}^{-1} \mathbf{Q}_{\hat{\boldsymbol{b}} \hat{\boldsymbol{z}}}^{T}
\end{aligned}
$$

\section{B. Integer Aperture Bootstrapping}

Integer Aperture Bootstrapping (IAB) [6] extends the IB concept to create a validation test that computes its test statistic using the ambiguity residual $\breve{\epsilon}$. The test statistic and decision criteria for IAB can be expressed as a function of the ambiguity residual and the aperture parameter $\beta \in[0,1]$ :

$$
T(\breve{\boldsymbol{\epsilon}}, \beta)=\left\|\operatorname{IB}\left(\frac{1}{\beta} \breve{\boldsymbol{\epsilon}}\right)\right\|_{0}
$$

where $\|\boldsymbol{v}\|_{0}=\left|\left\{i \mid v_{i} \neq 0\right\}\right|$ is the number of non-zero elements in the vector $\boldsymbol{v}$.

The IAB decision rule can be written as

$$
\delta(\breve{\boldsymbol{\epsilon}}, \beta)=\left\{\begin{array}{lll}
1 & \text { if } & T(\breve{\boldsymbol{\epsilon}}, \beta)=0 \\
0 & \text { if } & T(\breve{\boldsymbol{\epsilon}}, \beta) \neq 0
\end{array}\right.
$$

where the value of $\delta$ indicates whether the fix is accepted (1) or rejected $(0)$. In the event that the fix is rejected, IAB resorts 
to the float solution $\hat{\boldsymbol{x}}$. Note that a float or a fixed solution can be forced by choosing $\beta \in\{0,1\}$, since on this domain, $\delta(\breve{\boldsymbol{\epsilon}}, \beta)=\beta$.

The IAB decision rule can be interpreted as follows: If the IB algorithm is applied to a scaled-up version of the ambiguity residual and the result is the zero vector, then the ambiguity fix is accepted; otherwise, the fix is rejected. The IAB decision rule can be viewed as equivalent to that of IB but with a pullin region (PIR) shrunken by a factor of $\beta$, as illustrated in Fig. 1. The PIR is the region in $\mathbb{R}^{n}$ around the integer vector $\breve{z}$ for which the estimator returns $\breve{z}$ as its result.

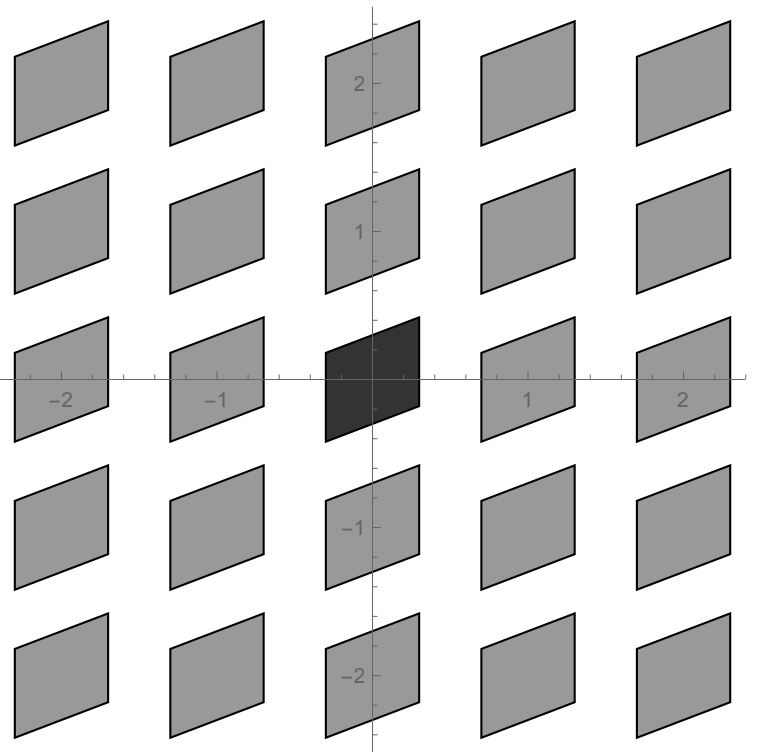

Fig. 1. Critical aperture regions for integer aperture bootstrapping for twodimensional example covariance model. This is a visual representation of the results of IAB when applied to $\boldsymbol{\epsilon}=\hat{\boldsymbol{z}}-\boldsymbol{z}$. The central, darkly shaded region is the success region, in which $\breve{z}=\boldsymbol{z}$. The lightly shaded regions correspond to incorrect ambiguity fixes, in which $\breve{\boldsymbol{z}} \neq \boldsymbol{z}$. When the ambiguity residual is in the unshaded region, the fix is rejected.

Figure 1 shows three important regions that correspond to three possible outcomes for an IA estimator. The central, dark region corresponds to the event defined as "success" in which the full ambiguity set is resolved without error. The union of the many lightly shaded regions correspond to the "failure" event in which one or more of the integer ambiguities is fixed incorrectly. Values of $\epsilon$ in the unshaded region results in the fix being rejected. This event is called "undecided." The probabilities of the events are reproduced from [6]:

$$
\begin{aligned}
& P_{F}=\sum_{\boldsymbol{z} \in \mathbb{Z}^{n} \backslash\{0\}} \prod_{i=1}^{n}\left(\Phi\left(\frac{\frac{\beta}{2}-L_{i}^{-1} \boldsymbol{z}}{\sigma_{i \mid I}}\right)-\Phi\left(\frac{-\frac{\beta}{2}-L_{i}^{-1} \boldsymbol{z}}{\sigma_{i \mid I}}\right)\right) \\
& P_{S}=\prod_{i=1}^{n}\left(2 \Phi\left(\frac{\beta / 2}{\sigma_{i \mid I}}\right)-1\right) \\
& P_{U}=1-P_{F}-P_{S}
\end{aligned}
$$

with $\beta$ as define previously and

$$
\begin{aligned}
& L_{i}^{-1}: \text { The } i^{\text {th }} \text { row of the } L^{-1} \text { matrix from the } \\
& \text { decomposition of the float covariance matrix } \\
& \sigma_{i \mid I}=\sqrt{d_{i}} \text { : The conditional standard deviation } \\
& \text { of the float ambiguity } \\
& \Phi(\cdot) \text { : The CDF of the standard normal random variable }
\end{aligned}
$$

A few important observations should be made about the event probabilities. First, the probability of failure, $P_{F}$, involves an infinite sum over all integer ambiguities other than the correct ambiguity. This can be approximated by summing over a large number of alternative ambiguities, but this may still be computationally expensive if the desired $P_{F}$ is small or if $n_{\mathrm{z}}$ is large. Second, $P_{F}$ is a monotonically increasing function of $\beta$, which implies that the risk of failure decreases as the integer aperture is made smaller. Thus, the aperture parameter $\beta$ controls the exposure to failure risk. Finally, $P_{S}$ is also monotonically increasing in $\beta$. This implies that any increase in $P_{S}$ will be at the expense of an increase in $P_{F}$ for this algorithm.

\section{Generalization to Partial Ambiguity Resolution}

To generalize IAB to partial ambiguity resolution, each ambiguity is fixed and validated successively by examining the magnitude of its conditional ambiguity residual. Since each ambiguity has a different conditional variance, $d_{i}$, each test is allowed a different $\beta_{i}$. The benefits of element specific $\beta_{i}$ are shown in section IV-B. The number of ambiguity fixes which pass the validation test is denoted as $n_{\mathrm{fix}}$. The algorithm, called GIAB is given in pseudocode as

$$
\begin{aligned}
& n_{\mathrm{fix}}=0 \\
& \text { for } i=1: n_{\mathrm{z}} \\
& \quad \hat{z}_{\mathrm{c}, i}=\hat{z}_{i}-\sum_{j=1}^{i-1} l_{i j} \breve{\epsilon}_{\mathrm{c}, j} \\
& \breve{z}_{i}=\left\lfloor\hat{z}_{\mathrm{c}, i}\right\rceil \\
& \breve{\epsilon}_{\mathrm{c}, i}=\hat{z}_{\mathrm{c}, i}-\breve{z}_{i} \\
& \text { if } \breve{\epsilon}_{\mathrm{c}, i}<\frac{\beta_{i}}{2} \\
& \quad n_{\mathrm{fix}}=i \\
& \text { else } \\
& \quad \text { break } \\
& \text { end } \\
& \text { end }
\end{aligned}
$$

In the event that $n_{\mathrm{fix}}<n_{\mathrm{z}}$, the next ambiguity, $i=n_{\mathrm{fix}}+$ 1 , cannot be fixed without violating the specified value of $P_{F}$. The integrity implications of partial fixing are addressed in section III-A. For now, assume that $\hat{z}_{i}, i>n_{\mathrm{fix}}$ are left floating. This decision will be revisited in a later section.

GIAB reduces to IAB if all $\breve{z}_{i}$ are rejected when $n_{\text {fix }} \neq n_{\mathrm{z}}$. For GIAB, a reduced set of the integer ambiguities can be used. Instead of having three events of success, failure, and 
indecision, GIAB leads to $n_{\mathrm{z}}+2$ events that can be defined as the failure event $F=\{\breve{z} \neq \boldsymbol{z}\}$ and success events $S_{n}=$ $\left\{\wedge_{i=1}^{n}\left(\breve{z}_{i}=z_{i}\right) \wedge\left(n_{\text {fix }}=n\right)\right\}$ for $n \in\left\{0,1, \ldots, n_{\mathrm{z}}\right\}$. That is, failure is defined as the acceptance of any incorrect integers, and one of $n_{\mathrm{z}}+1$ success cases is defined for each possible number of integer fixes from 0 to $n_{\mathrm{z}}$, provided no ambiguity is fixed incorrectly.

\section{Partial Ambiguity Resolution Probabilities}

To predict algorithm performance, the probability of each possible event must be computed. For each integer that is reached by the GIAB algorithm, there are three possibilities: the fix is accepted correctly, the fix is rejected, or the fix is accepted erroneously. Conditioned on the event that $n_{\mathrm{fix}}=i-1$ and that for $n_{\mathrm{fix}}>1, \breve{z}_{j}=z_{j}, \forall j=1, \ldots, n_{\mathrm{fix}}$, these three event probabilities follow trivially from (11):

$$
\begin{aligned}
P_{C, i} & =P\left(\left|\epsilon_{\mathrm{c}, \mathrm{i}}\right|<\frac{\beta_{i}}{2}\right)=2 \Phi\left(\frac{\beta_{i} / 2}{\sigma_{i \mid I}}\right)-1 \\
P_{E, i} & =\sum_{\zeta \in \mathbb{Z} \backslash\{0\}} P\left(\left|\epsilon_{\mathrm{c}, \mathrm{i}}-\zeta\right|<\frac{\beta_{i}}{2}\right) \\
& =\sum_{\zeta \in \mathbb{Z} \backslash\{0\}}\left(\Phi\left(\frac{\frac{\beta_{i}}{2}-\zeta}{\sigma_{i \mid I}}\right)-\Phi\left(\frac{-\frac{\beta_{i}}{2}-\zeta}{\sigma_{i \mid I}}\right)\right) \\
P_{R, i} & =1-P_{E, i}-P_{C, i}
\end{aligned}
$$

A simple bound on $P_{E, i}$ can be constructed by recognizing that the probability of rejection, $P_{R, i}$ need not be tightly controlled. It can be shown that $P_{R, i}$ can be bounded below by $P\left(\frac{\beta_{i}}{2} \leq\left|\epsilon_{\mathrm{c}, \mathrm{i}}\right| \leq 1-\frac{\beta_{i}}{2}\right)$ since this the region $\frac{\beta_{i}}{2} \leq\left|\epsilon_{\mathrm{c}, \mathrm{i}}\right| \leq$ $1-\frac{\beta_{i}}{2}$ is a subset of the rejection region. This leads to an upper bound on $P_{E, i}$ :

$$
\begin{aligned}
& P_{R, i} \geq 1-2 \Phi\left(\frac{\beta_{i} / 2-1}{\sigma_{i \mid I}}\right)-P_{C, i} \\
& P_{E, i} \leq 2 \Phi\left(\frac{\beta_{i} / 2-1}{\sigma_{i \mid I}}\right)
\end{aligned}
$$

Using these bounds, the overall event probabilities can be obtained and bounded. A failure event can be caused by any single fixing error. If there are multiple errant fixes, there is still only a single failure event as defined earlier. As such, the failure probability upper bound can be computed as

$$
\begin{aligned}
P_{F} & =P_{E, 1}+\sum_{i=2}^{n_{\mathrm{z}}} P_{E, i} \prod_{j=1}^{i-1} P_{C, j} \\
& \leq 2 \Phi\left(\frac{\beta_{i} / 2-1}{\sigma_{1}}\right) \\
& +\sum_{i=2}^{n_{\mathrm{z}}}\left(2 \Phi\left(\frac{\beta_{i} / 2-1}{\sigma_{i \mid I}}\right)\right) \prod_{j=1}^{i-1}\left(2 \Phi\left(\frac{\beta_{j} / 2}{\sigma_{j \mid J}}\right)-1\right)
\end{aligned}
$$

The probability of successfully fixing any number of integers can be computed similarly. For the probability of correctly resolving all ambiguities, the expression is equal to the probability of success $P_{S}$, as defined for IAB. For

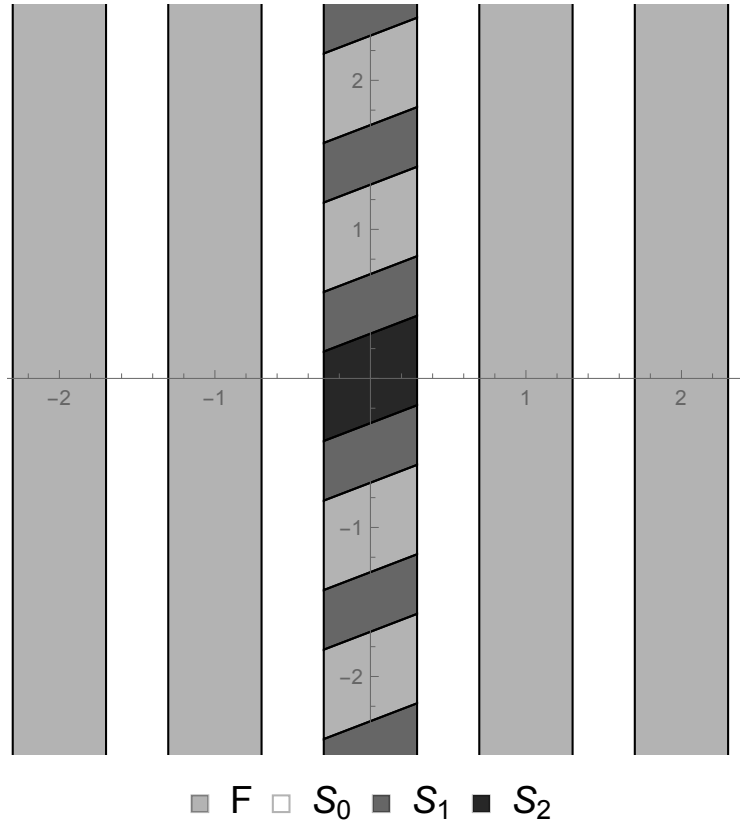

Fig. 2. Pull-in regions and associated events for GIAB. Event $F$ results if one or more of the ambiguities is fixed incorrectly. Events $S_{i}$ occur when exactly $i$ ambiguities are fixed and all ambiguities are fixed correctly.

GIAB, this probability is denoted as $P_{S_{n_{z}}}$. The remaining probabilities are

$$
\begin{aligned}
P_{S_{i}} & =P_{R, i+1} \prod_{j=1}^{i-1} P_{C, j} \\
& \geq 2\left(\Phi\left(\frac{-\beta_{i} / 2}{\sigma_{i \mid I}}\right)-\Phi\left(\frac{\beta_{i} / 2-1}{\sigma_{i \mid I}}\right)\right) \\
& \times \prod_{j=1}^{i-1}\left(2 \Phi\left(\frac{\beta_{j} / 2}{\sigma_{j \mid J}}\right)-1\right), i=1, \ldots, n_{z} \\
P_{S_{0}} & =P_{R, 1} \\
& \geq 2\left(\Phi\left(\frac{-\beta_{i} / 2}{\sigma_{1}}\right)-\Phi\left(\frac{\beta_{i} / 2-1}{\sigma_{1}}\right)\right)
\end{aligned}
$$

As long as the allowable $P_{F}$ is small, these bounds will be quite tight. The PIRs corresponding to these events are illustrated in Fig. 2 for $n_{\mathrm{z}}=2$.

\section{Prior And Posterior Distribution of GENERALIZED INTEGER APERTURE BASELINE}

To assess the performance of the GIAB algorithm, both the a priori and a posteriori distributions must be characterized. These distributions will be developed in a three step process. First, the a posteriori distribution will be developed when conditioned on $\wedge_{j=1}^{i}\left(\breve{z}_{j}=z_{j}\right) \wedge\left(n_{\mathrm{fix}}=i\right) \wedge\left(\epsilon_{\mathrm{c}, \mathrm{i}+1}=\varepsilon\right) \wedge$ $\left(\frac{\beta_{i+1}}{2}<|\varepsilon|<\frac{1}{2}\right)$; i.e., conditioned on the event that the first $n_{\mathrm{fix}}=i$ fixes were correct, and on the value of the $i+1^{s t}$ conditional ambiguity residual failed the threshold test. Next, the distribution of the partially fixed baseline conditioned only on the number of ambiguities fixed by GIAB will be derived. Finally, the a priori distribution of GIAB will be developed 
by removing the conditioning on the number of integers fixed. The first distribution will be referred to as the posterior distribution. It is the distribution that must be used by a real-time implementation to bound the $I R$ of the solution. The second distribution will be called the intermediate distribution. It is a mathematical construct used to reach the third distribution, which is called the prior distribution. The prior distribution can be used to analyze the average performance of the algorithm. Mathematically, the (1) posterior, (2) intermediate, and (3) prior distributions can be described as

$$
\begin{aligned}
& \text { (1) } f_{\breve{b} \mid \varepsilon, i}(\boldsymbol{\zeta})=P\left(\breve{\boldsymbol{b}}=\boldsymbol{\zeta} \mid \breve{\epsilon}_{\mathrm{c}, i+1}=\varepsilon, n_{\mathrm{fix}}=i\right) \\
& (2) f_{\breve{b} \mid i}(\boldsymbol{\zeta})=\int f_{\breve{b} \mid \varepsilon, i}(\boldsymbol{\zeta}) P\left(\breve{\epsilon}_{\mathrm{c}, i+1}=\varepsilon \mid n_{\mathrm{fix}}=i\right) d \varepsilon \\
& (3) f_{\breve{b}}(\boldsymbol{\zeta})=\sum_{i=0}^{n_{\mathrm{z}}} f_{\breve{\boldsymbol{b}}, i}(\boldsymbol{\zeta}) P\left(n_{\mathrm{fix}}=i\right)
\end{aligned}
$$

\section{A. The Posterior Distribution}

As a first step toward developing the a posteriori baseline error distribution, consider the event $S_{i}$ in terms of the values of the conditional ambiguity residuals, $\breve{\epsilon}_{\mathrm{c}}$, and the aperture threshold value, $\beta$. Assuming that $\beta$ has been set to control $P_{F}$ to an acceptable level, whenever the conditional ambiguity residual exceeds the threshold, that integer cannot be fixed without incurring an unacceptable risk. Further, if the bound in equation (16) is used, the risk of the incorrect fix being wrong by more than 1 is negligible. In equation form

$$
\begin{aligned}
& \text { Event } S_{i} \Rightarrow \\
& \qquad\left(\left|\breve{\epsilon}_{\mathrm{c}, j}\right|<\frac{\beta_{j}}{2}, \forall j \leq i\right) \wedge\left(\frac{\beta_{i+1}}{2}<\left|\breve{\epsilon}_{\mathrm{c}, i+1}\right|<1-\frac{\beta_{i+1}}{2}\right)
\end{aligned}
$$

According to the partial fixing procedure, the float baseline will have been conditioned upon the first $n_{\mathrm{fix}}=i$ ambiguities. For a particular value of $\breve{\epsilon}_{\mathrm{c}, i+1}$ that exceeds the threshold, the conditional maximum a posteriori ambiguity fix is that produced by integer bootstrapping, $\breve{z}_{i+1}$. However, this integer fix cannot be assumed without violating the probability of failure. The next most likely fix and its associated conditional ambiguity residual are

$$
\begin{aligned}
\breve{z}_{\text {alt }, i+1} & =\breve{z}_{i+1}+\operatorname{sign}\left(\breve{\epsilon}_{\mathrm{c}, i+1}\right) \\
\breve{\epsilon}_{\mathrm{c}, \text { alt }, i+1} & =\breve{\epsilon}_{\mathrm{c}, i+1}-\operatorname{sign}\left(\breve{\epsilon}_{\mathrm{c}, i+1}\right)
\end{aligned}
$$

To assess the effect of the unfixed residual upon the $a$ posteriori distribution, consider the following derivation of the fixed baseline adjustment using the conditional ambiguity residual instead of the usual method, which uses the unconditional ambiguity residual. Because there is a triangular linear transformation between the conditional and unconditional ambiguity residuals, $\breve{\epsilon}=L \breve{\epsilon}_{\mathrm{c}}$, conditioning upon the same subset of elements from 1 to $n_{\text {fix }}$ of $\breve{\boldsymbol{\epsilon}}$ or $\breve{\epsilon}_{\mathrm{c}}$ yields an equivalent result. Let $\mathbf{Q}_{\hat{\boldsymbol{b}} \hat{z}_{\mathrm{c}}}^{\mathcal{I}}$ denote the columns of the matrix $\mathbf{Q}_{\hat{\boldsymbol{b}} \hat{\boldsymbol{z}}_{\mathrm{c}}}$ indexed by a set of integers $\mathcal{I} \subseteq\left\{1,2,3, \ldots, n_{\mathrm{z}}\right\}$.

$$
\begin{aligned}
\breve{\boldsymbol{b}} & =\hat{\boldsymbol{b}}-\mathbf{Q}_{\hat{\boldsymbol{z}} \hat{\boldsymbol{z}}} \mathbf{Q}_{\hat{\boldsymbol{z}}}^{-1}(\hat{\boldsymbol{z}}-\breve{\boldsymbol{z}}) \\
& =\hat{\boldsymbol{b}}-\mathbf{Q}_{\hat{\boldsymbol{b}} \hat{\boldsymbol{z}}}\left[L D L^{T}\right]^{-1} \breve{\boldsymbol{\epsilon}} \\
& =\hat{\boldsymbol{b}}-\mathbf{Q}_{\hat{\boldsymbol{b}} \hat{\boldsymbol{z}}}\left[L^{-T} D^{-1} L^{-1}\right]\left(L \cdot \breve{\boldsymbol{\epsilon}}_{\mathrm{c}}\right) \\
& =\hat{\boldsymbol{b}}-\underbrace{\left(\mathbf{Q}_{\hat{\boldsymbol{z}} \hat{\boldsymbol{z}}} L^{-T}\right)}_{\mathbf{Q}_{\hat{\boldsymbol{b}} \hat{z}_{\mathrm{c}}}} D^{-1} \breve{\boldsymbol{\epsilon}}_{\mathrm{c}} \\
& =\hat{\boldsymbol{b}}-\mathbf{Q}_{\hat{\boldsymbol{b}} \hat{\boldsymbol{z}}_{\mathrm{c}}}\left[\begin{array}{c}
\frac{\breve{\epsilon}_{\mathrm{c}, 1}}{d_{1}} \\
\frac{\epsilon_{c, 2}}{d_{2}} \\
\vdots \\
\frac{\breve{\epsilon}_{\mathrm{c}, n_{z}}}{d_{n_{\mathrm{z}}}}
\end{array}\right] \\
& =\hat{\boldsymbol{b}}-\sum_{i=1}^{n_{\mathrm{z}}} \mathbf{Q}_{\hat{\boldsymbol{b}} \hat{\boldsymbol{z}}_{\mathrm{c}}}^{\{i\}} \frac{\breve{\epsilon}_{\mathrm{c}, i}}{d_{i}}
\end{aligned}
$$

By conditioning the fixed baseline solution using the sequentially-conditioned ambiguity residuals, the successive corrections can be performed simply, and without inverting the ambiguity matrix repeatedly for each correction. This expression implies that if the $i+1^{s t}$ integer were to be fixed, the adjustments in the most likely and alternate cases would be

$$
\begin{gathered}
\breve{\boldsymbol{b}}_{n_{\mathrm{fix}}=i+1}-\breve{\boldsymbol{b}}_{n_{\mathrm{fix}}=i+1}=-\mathbf{Q}_{\hat{\boldsymbol{b}} \hat{\boldsymbol{z}}_{\mathrm{c}}}^{\{i\}} \frac{\breve{\epsilon}_{\mathrm{c}}, i+1}{d_{i+1}} \\
\breve{\boldsymbol{b}}_{n_{\mathrm{fix}}=i+1, \mathrm{alt}}-\breve{\boldsymbol{b}}_{n_{\mathrm{fix}}=i+1}=-\mathbf{Q}_{\hat{\boldsymbol{b}} \boldsymbol{z}_{\mathrm{c}}}^{\{i\}} \frac{\breve{\epsilon}_{\mathrm{c}, \text { alt }, i+1}}{d_{i+1}}
\end{gathered}
$$

This expression shows that, in general, there will be a position domain bias in the baseline computed by leaving the $i+1^{s t}$ ambiguity unfixed. That is, if only $i$ ambiguities are fixed with $i<n_{\mathrm{z}}$, it is an invalid-and potentially hazardous - assumption that the resulting solution is unbiased. The effect of these biases must be addressed in the position domain if the integrity of the CDGPS solution is to be protected.

This fact motivates at least three variations of GIAB that differ only in how the ambiguity that exceeds the aperture threshold is handled. The first variation, termed float GIAB, will leave the unsatisfactory ambiguity as a real-valued estimate. This is the typical practice in the existing literature, but in float GIAB, the integrity risk due to the residual bias is explicitly taken into account. The second variation, called maximum a posteriori (MAP) GIAB, will accept the most likely fix candidate, but integrity risk from the alternate fix and its position domain bias is monitored. This is similar to the concept of position domain integrity in the EPIC and GERAFS algorithms, but there is no need to search a large set of alternative fixes since there is only a single non-negligible alternative fix candidate.

The third variation is minimum mean square error (MMSE) GIAB. In MMSE GIAB, a weighted average of the MAP GIAB solution and the alternative fixed solution is computed. When the MAP fix is much more likely than the alternative, MMSE GIAB will differ only slightly from the MAP GIAB solution. In the unlikely event that the two candidate fixes are 
equally likely, the MMSE GIAB baseline will equal the float GIAB baseline.

1) Position Domain Integrity in EPIC: For position domain integrity monitoring, the probability of large position domain error must be bounded, even in the event of incorrect fixes. For each of the components of the baseline vector, e.g., vertical, there will be an $A L$ that must bound the solution error to a specified level of integrity risk $\left(I R_{\text {spec }}\right)$. The risk that the $A L$ will be exceeded must be monitored to ensure that

$$
R=P(|\breve{b}-b| \geq A L)<I R_{\text {spec }}
$$

EPIC protects solution integrity by evaluating the $I R$ from the case that the ambiguities are fixed correctly and from a set of alternative fixes of size $n_{\text {alt }}$. Define $\mathcal{E}_{0}$ to be the event that the chosen ambiguity fix is correct. Define $\mathcal{E}_{j}$ to be the event that the $j^{\text {th }}$ alternative fix is correct (in which case the fix used in the solution is incorrect). Define $\mathcal{E}_{\infty}$ as the event that the correct fix was neither the chosen fix, nor among the alternative fixes. Let $R_{j}$ be the conditional risk of excess error given the event $\mathcal{E}_{j}$. The total risk is then

$$
R=R_{\infty} P\left(\mathcal{E}_{\infty}\right)+\sum_{j=0}^{n_{\text {alt }}} R_{j} P\left(\mathcal{E}_{j}\right)
$$

A bound on the risk of excess error can be derived by assuming that any incorrect fix not examined will cause excessive error, i.e., $R_{\infty}=1$. This leads to the bound used by EPIC to monitor risk of excessive error

$$
\begin{aligned}
R & \leq R_{\infty}+\sum_{i=0}^{n_{\text {alt }}} R_{j} P\left(\mathcal{E}_{j}\right) \\
& \leq 1-\sum_{j=0}^{n_{\text {alt }}} P\left(\mathcal{E}_{j}\right)+\sum_{j=0}^{n_{\text {alt }}} R_{j} P\left(\mathcal{E}_{j}\right)
\end{aligned}
$$

In the EPIC algorithm, the event probabilities are the $a$ priori fixing probabilities for integer bootstrapping

$$
\begin{aligned}
& P\left(\mathcal{E}_{j}\right)= \\
& \quad \prod_{k=1}^{n}\left(\Phi\left(\frac{\frac{1}{2}-L_{k}^{-1} \cdot \boldsymbol{z}_{j}[k]}{\sigma_{k \mid K}}\right)-\Phi\left(\frac{-\frac{1}{2}-L_{k}^{-1} \cdot \boldsymbol{z}_{j}[k]}{\sigma_{k \mid K}}\right)\right)
\end{aligned}
$$

The conditional risk, assuming zero-mean Gaussian measurements, results from the position domain bias induced by incorrect ambiguity fixing, $\mu_{j}$ and the variance of the component of the fixed baseline being protected, $\sigma_{\breve{b}}^{2}$

$$
R_{j}=\Phi\left(\frac{A L-\mu_{j}}{\sigma_{\breve{b}}}\right)-\Phi\left(\frac{-A L-\mu_{j}}{\sigma_{\breve{b}}}\right)
$$

2) Position Domain Integrity in GIAB: For GIAB, the differences from EPIC are that the event probabilities are the a posteriori fixing probabilities instead of the a priori, that there is only a single alternative fix that must be considered, and that there will be a bias in the most likely case if float GIAB or MMSE GIAB are used. For each of the three versions of GIAB, there will be two primary models and a third model to account for the probability of incorrect fix. This means that the distributions can be modeled as Gaussian mixture models

$$
f_{\breve{b} \mid \varepsilon, i}(\zeta)=\sum_{j=0}^{2} P\left(\mu=\mu_{j} \mid \varepsilon, i\right) \mathcal{N}\left(\zeta ; \mu_{j}, \mathbf{Q}_{\breve{b} \mid \mathcal{I}+1}\right)
$$

where $\mathbf{Q}_{\breve{b} \mid \mathcal{I}+1}$ is the covariance of the fixed baseline when conditioned on the first $i+1$ integer ambiguities.

The modal biases $\mu_{0}$ and $\mu_{1}$ result from the $i+1^{\text {st }}$ ambiguity, while $\mu_{2}$ is set arbitrarily large to reflect the uncertain result of an incorrect fix. The three models all have the same covariance since they all result from conditioning on the same set of ambiguities. The covariance of the baseline conditioned upon these ambiguities is computed using the submatrices of the $\mathbf{Q}_{\hat{\boldsymbol{b}}, \hat{\boldsymbol{z}}_{c}}$ and $D$ matrices that correspond to those ambiguities. Let $\mathcal{I}+1$ be the set of indices from 1 to $i+1$. The matrix $D^{\mathcal{I}+1, \mathcal{I}+1}$ is the submatrix of $D$ corresponding to these indices.

$$
\mathbf{Q}_{\breve{b} \mid \mathcal{I}+1}=\mathbf{Q}_{\hat{\boldsymbol{b}}}-\mathbf{Q}_{\hat{\boldsymbol{b}}, \hat{\boldsymbol{z}}_{c}}^{\mathcal{I}+1}\left(D^{\mathcal{I}+1, \mathcal{I}+1}\right)^{-1}\left(\mathbf{Q}_{\hat{\boldsymbol{b}}, \hat{\boldsymbol{z}}_{c}}^{\mathcal{I}+1}\right)^{T}
$$

The risk bound for GIAB can be simplified from that of EPIC since $n_{\text {alt }}=1$ for GIAB.

$$
R \leq 1-\left(1-R_{0}\right) P\left(\mathcal{E}_{0}\right)-\left(1-R_{1}\right) P\left(\mathcal{E}_{1}\right)
$$

To bound the mode probabilities $P\left(\mu=\mu_{j} \mid \varepsilon, i\right)$, which correspond to the event probabilities in EPIC, $P\left(\mathcal{E}_{j}\right)$, apply Bayes rule to the fixing probabilities of the $i+1^{\text {st }}$ ambiguity $P\left(z_{i+1}=\breve{z}_{j} \mid \varepsilon, i\right)$. Since the biases correspond to integer values that are unknown, assume a diffuse prior on the integers; i.e., the a priori probabilities for each ambiguity vector are equally likely. This diffuse prior should not be confused with the prior probability that integer bootstrapping will find the correct integer, whatever it may be, which was given in equation (26).

$$
P\left(z_{i+1}=\breve{z}_{i+1} \mid \varepsilon, i\right)=\frac{P\left(\varepsilon \mid z_{i+1}=\breve{z}_{i+1}, n_{\mathrm{fix}}=i\right)}{\sum_{k \in \mathbb{Z}} P\left(\varepsilon \mid z_{i+1}=\breve{z}_{i+1}+k, n_{\mathrm{fix}}=i\right)}
$$

In order to make a bounding argument, let

$$
\begin{aligned}
\lambda_{0} & =P\left(\varepsilon \mid z_{i+1}=\breve{z}_{i+1}, n_{\mathrm{fix}}=i\right) \\
\lambda_{1} & =P\left(\varepsilon \mid z_{i+1}=\breve{z}_{\mathrm{alt}, i+1}, n_{\mathrm{fix}}=i\right) \\
\lambda_{\infty} & =P\left(\varepsilon \mid z_{i+1} \notin\left\{\breve{z}_{\mathrm{alt}, i+1}, \breve{z}_{i+1}\right\}, n_{\mathrm{fix}}=i\right)
\end{aligned}
$$

Then

$$
\begin{aligned}
& p_{0}=P\left(z_{i+1}=\breve{z}_{i+1} \mid \varepsilon, i\right)=\frac{\lambda_{0}}{\lambda_{0}+\lambda_{1}+\lambda_{\infty}} \\
& p_{1}=P\left(z_{i+1}=\breve{z}_{\text {alt }, i+1} \mid \varepsilon, i\right)=\frac{\lambda_{1}}{\lambda_{0}+\lambda_{1}+\lambda_{\infty}}
\end{aligned}
$$

Because $\left\{\mathcal{E}_{0}, \mathcal{E}_{1}, \mathcal{E}_{\infty}\right\}$ form a partition, $p_{0}+p_{1}+p_{F}=$ 1 , where $p_{F}$ corresponds to the actual probability the $z \notin$ 
$\left\{\breve{z}_{0}, \breve{z}_{1}\right\}$. If this quantity were known precisely, the value of $\lambda_{\infty}$ could be computed in terms of $\lambda_{0}$ and $\lambda_{1}$ as follows

$$
\begin{aligned}
& p_{0}+p_{1}=1-p_{F} \\
& \frac{\lambda_{0}}{\lambda_{0}+\lambda_{1}+\lambda_{\infty}}+\frac{\lambda_{1}}{\lambda_{0}+\lambda_{1}+\lambda_{\infty}}=1-p_{F} \\
& \frac{\lambda_{0}+\lambda_{1}}{\lambda_{0}+\lambda_{1}+\lambda_{\infty}}=1-p_{F} \\
& \lambda_{\infty}=\frac{p_{F}}{1-p_{F}}\left(\lambda_{0}+\lambda_{1}\right)
\end{aligned}
$$

Some simple algebraic manipulation gives the result

$$
p_{j}=\frac{\lambda_{j}}{\lambda_{0}+\lambda_{1}}\left(1-p_{F}\right)
$$

Because zero-mean Gaussian errors are assumed, the fixing probabilities can be further simplified. Note that under the given conditions, $\left(z_{i+1}=\breve{z}_{i+1}\right) \Rightarrow\left(\varepsilon=\breve{\epsilon}_{\mathrm{c}, i+1}\right)$ and $\left(z_{i+1}=\breve{z}_{\mathrm{alt}, i+1}\right) \Rightarrow\left(\varepsilon=\breve{\epsilon}_{\mathrm{c}, i+1}-\operatorname{sign}\left(\breve{\epsilon}_{\mathrm{c}, i+1}\right)\right)$.

$$
\begin{aligned}
p_{0} & =\frac{\exp \left\{-\frac{\breve{\epsilon}_{,, i+1}^{2}}{2 d_{i+1}}\right\}\left(1-p_{F}\right)}{\exp \left\{-\frac{\breve{\epsilon}_{\mathrm{c}, i+1}^{2}}{2 d_{i+1}}\right\}+\exp \left\{-\frac{\left(\breve{\epsilon}_{\mathrm{c}, i+1}-\operatorname{sign}\left(\breve{\epsilon}_{\mathrm{c}, i+1}\right)\right)^{2}}{2 d_{i+1}}\right\}} \\
= & \frac{\left(1-p_{F}\right)}{1+\exp \left\{\frac{\left(\breve{\epsilon}_{\mathrm{c}, i+1}\right)^{2}}{2 d_{i+1}}-\frac{\left(\breve{\epsilon}_{\mathrm{c}, i+1}-\operatorname{sign}\left(\breve{\epsilon}_{\mathrm{c}, i+1}\right)\right)^{2}}{2 d_{i+1}}\right\}} \\
= & \frac{\left(1-p_{F}\right)}{1+\exp \left\{-\frac{1-2\left|\breve{\epsilon}_{\mathrm{c}, i+1}\right|}{2 d_{i+1}}\right\}}
\end{aligned}
$$

where $d_{i+1}$ is the $i+1^{\text {st }}$ element of the $D$ matrix.

Because $p_{0}+p_{1}=1-p_{F}$, the probability that the alternative fix is correct is simple to compute

$$
\begin{aligned}
p_{1} & =1-p_{F}-p_{0} \\
& =1-p_{F}-\frac{\left(1-p_{F}\right)}{1+\exp \left\{-\frac{1-2\left|\breve{\epsilon}_{\mathrm{c}, i+1}\right|}{2 d_{i+1}}\right\}} \\
& =\left(1-p_{F}\right)\left(1-\frac{1}{1+\exp \left\{-\frac{1-2\left|\breve{\epsilon}_{\mathrm{c}, i+1}\right|}{2 d_{i+1}}\right\}}\right) \\
& =\frac{\left(1-p_{F}\right)}{1+\exp \left\{\frac{1-2\left|\breve{\epsilon}_{\mathrm{c}, i+1}\right|}{2 d_{i+1}}\right\}}
\end{aligned}
$$

Because $\frac{\beta}{2} \leq\left|\breve{\epsilon}_{\mathrm{c}, i+1}\right| \leq \frac{1}{2}$, the fixing probabilities obey the inequality

$$
1-p_{F}<p_{1} \leq \frac{1-p_{F}}{2} \leq p_{0}<1-p_{F}
$$

By substituting equations (28), (35), and (36) into (23) with $\Phi(\cdot)$ the CDF of the standard normal distribution and $\breve{b}$ the component of $\vec{b}$ of interest, the a posteriori risk of excessive error

$$
\begin{aligned}
R_{b \mid \epsilon, i} \leq p_{F} & \\
+ & +p_{0}\left[1-\left(\Phi\left(\frac{A L-\mu_{1}}{\sigma_{\breve{b}, i+1}}\right)-\Phi\left(\frac{-A L-\mu_{1}}{\sigma_{\breve{b}, i+1}}\right)\right)\right] \\
& +p_{1}\left[1-\left(\Phi\left(\frac{A L-\mu_{2}}{\sigma_{\breve{b}, i+1}}\right)-\Phi\left(\frac{-A L-\mu_{2}}{\sigma_{\breve{b}, i+1}}\right)\right)\right]
\end{aligned}
$$

It is simple to show that (38) is monotonically increasing in $p_{F}$. Monotonicity allows the risk $R_{b \mid \epsilon, i}$ to be bounded by replacing $p_{F}$ with any upper bound, including $P_{F}$ from (16). A slightly tighter bound could be obtained by only including the failure risk for the first $i+1$ ambiguities since the other ambiguities remain floating.

$$
\begin{aligned}
P_{F, i+1} & \leq 2 \Phi\left(\frac{\beta_{1} / 2-1}{\sigma_{1}}\right) \\
& +\sum_{j=2}^{i+1}\left(2 \Phi\left(\frac{\beta_{j} / 2-1}{\sigma_{j \mid J}}\right)\right) \prod_{k=1}^{j-1}\left(2 \Phi\left(\frac{\beta_{k} / 2}{\sigma_{k \mid K}}\right)-1\right)
\end{aligned}
$$

All that remains is to determine the biases of the three solution varieties. For the float GIAB solution, the chosen solution is $\breve{\boldsymbol{b}}_{n_{\mathrm{fix}}=i}$, which implies that the biases can be computed as

$$
\begin{aligned}
\mu_{1, \text { float }} & =\mathbb{E}\left[\breve{\boldsymbol{b}}_{n_{\mathrm{fix}}=i}-\boldsymbol{b} \mid S_{i}, z_{i+1}=\breve{z}_{i+1}\right] \\
& =\mathbf{Q}_{\hat{\boldsymbol{b}} \hat{\boldsymbol{z}}_{\mathrm{c}}}^{\mathcal{I}+1} \frac{\breve{\epsilon}_{\mathrm{c}, i+1}}{d_{i+1}} \\
\mu_{2, \text { float }} & =\mathbb{E}\left[\breve{\boldsymbol{b}}_{n_{\mathrm{fix}}=i}-\boldsymbol{b} \mid S_{i}, z_{i+1}=\breve{z}_{\mathrm{alt}, i+1}\right] \\
& =\mathbf{Q}_{\hat{\boldsymbol{b}} \hat{\boldsymbol{z}}_{\mathrm{c}}}^{\mathcal{I}+1} \frac{\breve{\epsilon}_{\mathrm{c}, \text { alt }, i+1}}{d_{i+1}}
\end{aligned}
$$

Because $\frac{\beta_{i+1}}{2} \leq\left|\breve{\epsilon}_{\mathrm{c}, i+1}\right| \leq \frac{1}{2}$, equation (20b) implies that

$$
\begin{aligned}
& \operatorname{sign}\left(\mu_{2, \text { float }}\right)=-\operatorname{sign}\left(\mu_{1, \text { float }}\right) \\
& \left|\mu_{1, \text { float }}\right| \leq\left|\mu_{2, \text { float }}\right|
\end{aligned}
$$

For the MAP GIAB solution, the chosen solution is $\breve{\boldsymbol{b}}_{n_{\mathrm{fix}}=i+1}$, which is unbiased with probability $p_{0}$. This implies that

$$
\begin{aligned}
\mu_{1, \mathrm{MAP}} & =0 \\
\mu_{2, \mathrm{MAP}} & =\mu_{2, \text { float }}-\mu_{1, \text { float }} \\
& =-\mathbf{Q}_{\hat{\boldsymbol{b}} \hat{\boldsymbol{z}}_{\mathrm{c}}} \frac{\operatorname{sign}\left(\breve{\epsilon}_{\mathrm{c}, i+1}\right)}{d_{i+1}}
\end{aligned}
$$

The MMSE GIAB solution is formed by taking a weighted average of the MAP GIAB solution and the alternative solution. As such, its biases will be weighted averages of the MAP GIAB biases.

$$
\begin{aligned}
\breve{\boldsymbol{b}}_{\mathrm{MMSE}} & =\frac{p_{0}}{1-p_{F}} \breve{\boldsymbol{b}}_{n_{\mathrm{fix}}=i+1}+\frac{p_{1}}{1-p_{F}} \breve{\boldsymbol{b}}_{n_{\mathrm{fix}}=i+1, \text { alt }} \\
\mu_{1, \mathrm{MMSE}} & =-\frac{p_{1}}{1-p_{F}} \mu_{2, \mathrm{MAP}} \\
\mu_{2, \mathrm{MMSE}} & =\frac{p_{0}}{1-p_{F}} \mu_{2, \mathrm{MAP}}
\end{aligned}
$$

It is worth observing that the values of the means and risks of the three types of solutions follow particular inequalities. The typical accuracy of the algorithm is controlled by the value of $\mu_{1}$, while the risk $R_{b \mid \epsilon, i}$ is driven by the value of $\mu_{2}$. This observation, combined with the inequalities of (43) imply that MAP GIAB is typically the most accurate, but has the highest position domain $I R$ in the event of partial fixing. Also, float GIAB will typically be the least accurate, but have the lowest position domain $I R$ in the event of partial fixing. MMSE GIAB 


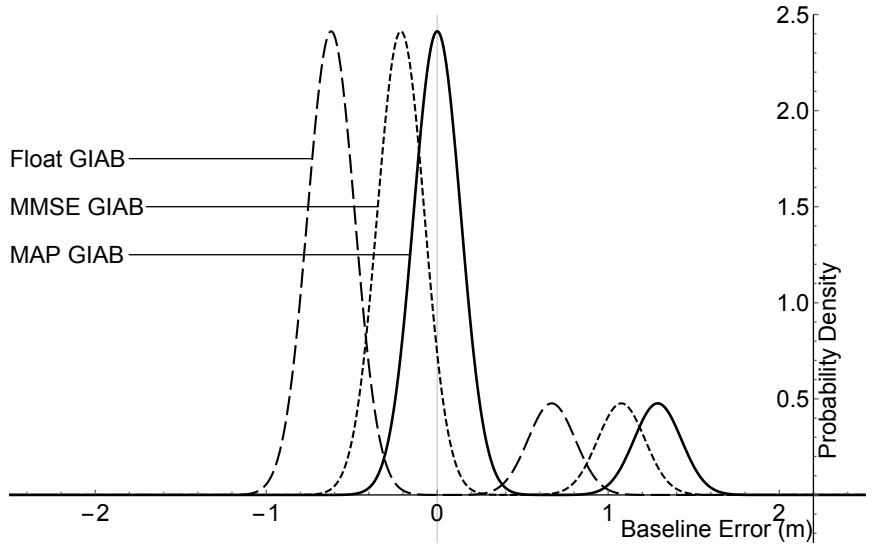

Fig. 3. Posterior distributions of one component of the partially fixed baseline error for Float, MAP, and MMSE GIAB for a large ambiguity residual with $n_{\mathrm{fix}}<n_{\mathrm{z}}$. Because the solutions differ by a bias as a function of the residual $\epsilon_{\mathrm{c}, i+1}$, the three distributions are shifted versions of each other, with that of MMSE GIAB between Float and MAP GIAB.

strikes the balance between the two solutions by favoring the most likely unbiased solution, but still including a weighted contribution of the next most likely solution. Depending on the relative importance and strictness of the accuracy and integrity requirements for the solution, any of the variations of GIAB may be preferred.

$$
\begin{aligned}
0=\mu_{1, \mathrm{MAP}} & <\left|\mu_{1, \mathrm{MMSE}}\right| \leq\left|\mu_{1, \text { float }}\right| \\
& \leq\left|\mu_{2, \text { float }}\right| \leq\left|\mu_{2, \mathrm{MMSE}}\right|<\left|\mu_{2, \mathrm{MAP}}\right|
\end{aligned}
$$

The posterior distributions of the errors in one dimension of the fixed baseline are plotted for the three variations of GIAB in Fig. 3. This example shows results from a very large $\breve{\epsilon}_{\mathrm{c}}[i+1]$ so that the MMSE and MAP distributions are visibly distinct. When the ambiguity residual is small, the MMSE and MAP distributions cannot be visibly distinguished and the secondary mode probabilities are too small to be seen on this scale.

\section{B. The Intermediate Distribution}

The intermediate distribution can now be computed by removing the conditioning upon the particular ambiguity residual. The region of integration is over $\frac{\beta}{2}<|\epsilon|<\frac{1}{2}$ since the magnitude of the ambiguity must be less than $\frac{1}{2}$, but has failed the threshold test. The probability density function is the Gaussain density function, normalized for the support of the event.

$$
\begin{aligned}
& P\left(\breve{\epsilon}_{\mathrm{c}, i+1}=\epsilon \mid n_{\mathrm{fix}}=i\right) \\
& =\frac{\phi\left(\frac{\varepsilon}{\sigma_{n+1 \mid N+1}}\right)\left(\mathbb{I}_{\left[-\frac{1}{2},-\frac{\beta_{i+1}}{2}\right]}+\mathbb{I}_{\left[\frac{\beta_{i+1}}{2}, \frac{1}{2}\right]}\right)}{2\left[\Phi\left(-\frac{\beta_{i+1}}{2 \sigma_{i+1 \mid I+1}}\right)-\Phi\left(-\frac{1}{2 \sigma_{i+1 \mid I+1}}\right)\right]}
\end{aligned}
$$

where:

$\mathbb{I}$ is the indicator function

$\phi(\cdot)$ is the PDF of the standard normal distribution

$\Phi$ is the CDF of the standard normal distribution
This expression can be substituted, along with equation (28) into the following integral to yield the PDF of the baseline estimate, conditioned on the number of integers that satisfied the aperture threshold test.

$$
f_{\breve{\boldsymbol{b}} \mid i}(\boldsymbol{\zeta} \mid i)=\int_{-\infty}^{\infty} f_{\breve{\boldsymbol{b}} \mid \varepsilon, i}(\boldsymbol{\zeta}) P\left(\breve{\epsilon}_{\mathrm{c}, i+1}=\varepsilon \mid n_{\mathrm{fix}}=i\right) d \varepsilon
$$

This integral is not able to be evaluated in closed form, but can be tightly bounded from a position domain integrity perspective by treating it as a mixture model of mixture models. The weights to be used when building this mixture model depend on the number of models that are being combined.

$$
\begin{aligned}
f_{\breve{b} \mid i}(\boldsymbol{\zeta}) & \approx \sum_{j=0}^{n_{\varepsilon}-1} w_{j}\left(f_{\breve{\boldsymbol{b}} \mid \varepsilon_{j}, i}(\boldsymbol{\zeta})+f_{\breve{\boldsymbol{b}} \mid-\varepsilon_{j}, i}(\boldsymbol{\zeta})\right) \\
w_{j} & =\frac{\left[\Phi\left(-\frac{1-2(j+1) \delta \varepsilon}{2 \sigma_{n+1 \mid N+1}}\right)-\Phi\left(-\frac{1-2(j) \delta \varepsilon}{2 \sigma_{i+1 \mid I+1}}\right)\right]}{2\left[\Phi\left(-\frac{\beta_{i+1}}{2 \sigma_{i+1 \mid I+1}}\right)-\Phi\left(-\frac{1}{2 \sigma_{i+1 \mid I+1}}\right)\right]} \\
\varepsilon_{j} & =-\frac{1}{2}+(j) \delta \varepsilon \\
\delta \varepsilon & =\frac{1-\beta_{i+1}}{2 n_{\varepsilon}}
\end{aligned}
$$

This expression is constructed by lumping the probability that $\breve{\epsilon}_{\mathrm{c}, i+1}=\varepsilon \in\left[\frac{1}{2}-(j+1) \delta \epsilon, \frac{1}{2}-(j+1) \delta \epsilon\right]$ with the largest magnitude residual in that region. This approach is conservative with respect to integrity because $p_{1}$ is monotonically increasing with $\breve{\epsilon}_{\mathrm{c}, i+1}$ for MAP GIAB, and $R_{b \mid \epsilon, i}$ is monotonically increasing with $p_{1}$. This implies that the computed risk will over bound the actual risk if the approximation in equation (46) is used. If $n_{\varepsilon}$ is allowed to be greater than 10 , the bound will be very tight. Since this distribution is only used for off-line analysis, its real-time performance is not critical. The intermediate distributions for float, MAP, and MMSE GIAB baselines are shown in Fig. 4.

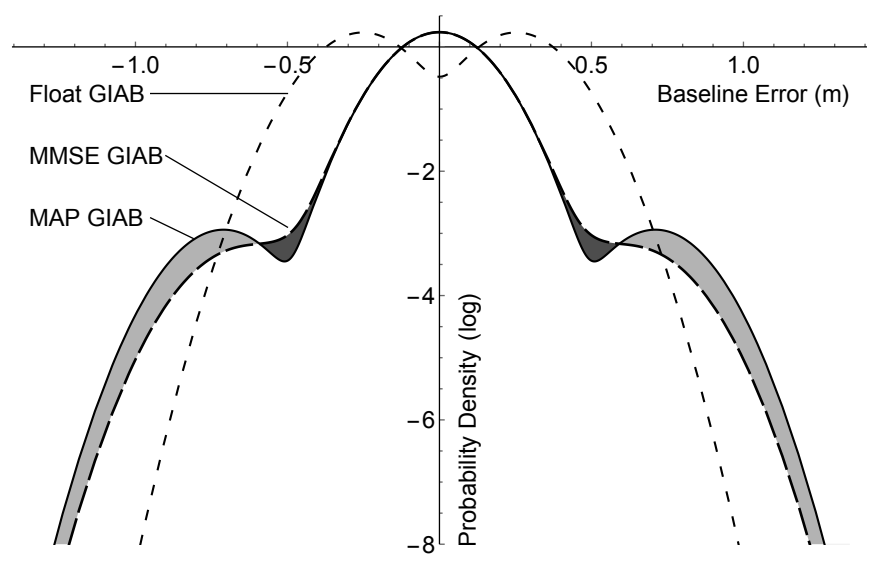

Fig. 4. Intermediate distributions of float, MAP, and MMSE GIAB for $n_{\mathrm{fix}}<$ $n_{\mathrm{z}}$, plotted in log scale. Float GIAB has a symmetric, bimodal distribution. MAP GIAB has a symmetric trimodal distribution with a dominant, zero-mean error central mode. MMSE GIAB has lower probabilities of large errors than does MAP GIAB at the expense of a slight increase in the probability of moderately sized errors. In the lightly shaded region, MAP GIAB has higher density. In the darker region, MMSE GIAB has higher density. 


\section{The Prior Distribution}

At this point, all the tools needed to construct the a priori distribution of the GIAB baseline are in place. Using the fixing probabilities of equations (16) and (17) together with the partially fixed covariance in equation (29) and the intermediate distribution in equation (46), the final a priori distribution is obtained in equation (47) on page 11.

This distribution is a Gaussian mixture model with a total of $4 n_{\mathrm{z}} n_{\varepsilon}+1$ models. There are 4 models for each ambiguity residual used for each number of partial fixes and a single mode for the correct fix case. This expression ignores the failure case, and so integrates to $1-P_{F}$.

For the MAP case, $\mu_{1, j}=0$, which implies that the unbiased models get double the weight and the resulting distribution has $3 n_{\mathrm{z}} n_{\varepsilon}+1$ mixed models. Since the strongest models are all zero-mean error, the MAP baseline has a much stronger unbiased mode than the Float GIAB baseline. This is illustrated in Fig. 5.

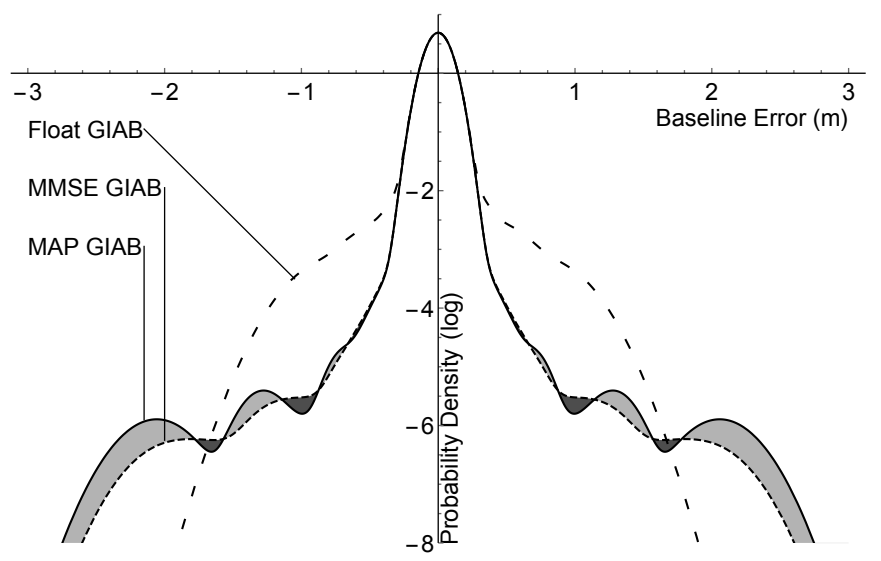

Fig. 5. Prior distributions of float, MAP, and MMSE GIAB, plotted in log scale. Float GIAB has a weak central mode with strong, but narrow secondary peaks. MAP and MMSE GIAB both have a strong central mode and secondary peaks that are wider, but weaker than those of float GIAB. MMSE GIAB has smoother and narrower secondary peaks than MAP GIAB.

\section{Setting the Integer Aperture Parameters}

\section{A. Allocation from Probability of Failure}

In IAB, a single threshold parameter $\beta$ is used to control the probability of failure with the probability of success falling out as an uncontrolled result. The fact that GIAB permits partial ambiguity resolution enables greater flexibility in setting the size and shape of the integer aperture than in IAB. If $n_{\mathrm{z}}$ different thresholds are set, this allows some control in the resulting values of $P_{S_{n_{\mathrm{Z}}}}$ for any desired $P_{F}$. Recall that $\beta_{i}$ be the threshold used to validate the $i^{\text {th }}$ integer ambiguity fix.

If $w_{i} P_{F}$ risk is allocated to incorrectly fixing the $i^{\text {th }}$ ambiguity, and if the preceding threshold values have been set, then $\beta_{i}$ can be set to maximize the probability of success by solving

$$
P_{E, i}\left(\beta_{i}\right)=\frac{w_{i} P_{F}}{\prod_{j=1}^{i-1} P_{C, j}}
$$

by inverting equation (15). The optimizing value of $\beta_{i}$ is

$$
\begin{aligned}
\beta_{i} & =\beta_{\max }\left(P_{F, i}, d_{i}, P_{C, j}\right) \\
& =\min \left[1, \max \left[0,2\left(1+\sqrt{d_{i}} \Phi^{-1}\left(\frac{P_{F, i}}{2 \prod_{j=1}^{i-1} P_{C, j}}\right)\right)\right]\right]
\end{aligned}
$$

If $\beta_{i}=0$ for any $i$, then the ambiguities cannot be fixed to the desired level of confidence. If $\beta_{i}=1$ for all $i$, then the fixed solution can be trusted to the desired level of confidence with full availability. The task of setting the aperture parameters has now been simplified to allocating the overall risk of incorrect fix to the individual ambiguities. The simplest way to set the aperture parameters is to use a bracketing iterative root finder with a single value for $\beta$. The initial values used in the search can be

$$
\begin{aligned}
& \beta_{\mathrm{LB}}=\beta_{\max }\left(\frac{P_{F}}{n_{\mathrm{z}}}, \max (D), 1\right) \\
& \beta_{\mathrm{UB}}=\beta_{\max }\left(\frac{P_{F}}{n_{\mathrm{z}}}, \min (D), 1\right)
\end{aligned}
$$

This approach effectively sets the aperture size by the ambiguity with the greatest uncertainty. It is conceivable that this will reduce the availability of the fixed solution since the rejection of an earlier ambiguity will terminate the GIAB fixing early. Further, since partial ambiguity resolution is being used, it may be advantageous to fix most of the ambiguities with higher availability than would be possible with a single fixed threshold. These reasons motivate the use of alternative allocations of the fixing risk. If an equal allocation of the overall specified failure risk is given to each ambiguity, the values are set as

$$
\beta_{i}=\beta_{\max }\left(\frac{P_{F}}{n_{\mathrm{z}}}, d_{i}, P_{C, j}\right)
$$

If some of the ambiguities are known with sufficient accuracy that $\beta_{i}=1$ for those values, then a further allocation can be made that adjusts the overall risk to be allocated to the remaining ambiguities. More generally, any allocation of risk to the individual parameters can be used that satisfies the following constraints:

$$
\begin{aligned}
\beta_{i}=\beta_{\max }\left(w_{i} P_{F}, d_{i}, P_{C, j}\right) \\
\text { s.t. } \quad \sum_{i=1}^{n_{\mathrm{z}}} w_{i}=1 \\
\quad 0<w_{i}<1, \forall i
\end{aligned}
$$

\section{B. Optimization for Availability of Full Ambiguity Resolution}

Given the constraints of (52) and a specified failure rate $P_{F \text {,spec }}$, maximize the percentage of time that the full ambiguity set can be resolved while satisfying the given constraints. Defining $A_{n}$ as the probability that $n$ or more integers were 


$$
\begin{aligned}
f_{\breve{\boldsymbol{b}}}(\boldsymbol{\zeta}) & =\sum_{i=0}^{n_{\mathrm{z}}} P_{S_{i}} f_{\breve{b} \mid i}(\boldsymbol{\zeta}) \\
& \approx P_{S_{n_{\mathrm{z}}}} f_{\breve{\boldsymbol{b}} \mid n_{\mathrm{z}}}(\boldsymbol{\zeta})+\sum_{i=0}^{n_{\mathrm{z}}-1} P_{S_{i}}\left\{\sum_{j=1}^{n_{\varepsilon}} w_{j}\left[f_{\breve{\boldsymbol{b}} \mid i, \varepsilon_{j}}(\boldsymbol{\zeta})+f_{\breve{\boldsymbol{b}} \mid i,-\varepsilon_{j}}(\boldsymbol{\zeta})\right]\right\} \\
& \approx P_{S_{n_{\mathrm{z}}}} \mathcal{N}\left(\boldsymbol{\zeta} ; \mathbf{0}, \mathbf{Q}_{\breve{\boldsymbol{b}}}\right)+\sum_{i=0}^{n_{\mathrm{z}}-1} P_{S_{i}} \sum_{j=1}^{n_{\varepsilon}} w_{j} \times\left\{\begin{array}{c}
p_{1, j} \mathcal{N}\left(\boldsymbol{\zeta} ; \boldsymbol{\mu}_{1, j}, \mathbf{Q}_{\breve{\boldsymbol{b}} \mid \mathcal{I}+1}\right)+p_{2, j} \mathcal{N}\left(\boldsymbol{\zeta} ; \boldsymbol{\mu}_{2, j}, \mathbf{Q}_{\breve{\boldsymbol{b}} \mid \mathcal{I}+1}\right) \\
+p_{1, j} \mathcal{N}\left(\boldsymbol{\zeta} ;-\boldsymbol{\mu}_{1, j}, \mathbf{Q}_{\breve{b} \mid \mathcal{I}+1}\right)+p_{2, j} \mathcal{N}\left(\boldsymbol{\zeta} ;-\boldsymbol{\mu}_{2, j}, \mathbf{Q}_{\breve{\boldsymbol{b}} \mid \mathcal{I}+1}\right)
\end{array}\right\}
\end{aligned}
$$

correctly fixed and none were incorrectly fixed, and using the events of (15)

$$
A_{n}=\prod_{i=1}^{n} P_{C, i} \prod_{j=n+1}^{n_{\mathrm{z}}}\left(1-P_{E, j}\right)
$$

The optimization problem can be posed as

$$
\begin{array}{ll} 
& \boldsymbol{\beta}^{*}=\underset{\boldsymbol{\beta}}{\arg \max }\left[A_{n_{\mathrm{z}}}(\boldsymbol{\beta})\right] \\
\text { s.t. } & P_{F}(\boldsymbol{\beta}) \leq P_{F, \text { spec }}
\end{array}
$$

This problem was approached using a gradient descent method, but since neither $P_{F}$ nor $A_{n_{a}}$ is a convex function of the vector of aperture parameters, $\boldsymbol{\beta}^{*} \in[0,1]^{n_{\mathrm{z}}}$, there are many local maxima in the region of the global maximum. Because there is no guarantee of finding the global optimum and the gradient is very computationally expensive, it is desirable to find a simple heuristic to compute the weights that results in high availability.

Since both $P_{F}$ and $A_{n}$ are functions of the conditional variances $d_{i}$, it is reasonable to compute the weights as functions of $d_{i}$ as well. That is

$$
w_{i}=\frac{f\left(d_{i}\right)}{\sum_{j=1}^{n_{\mathrm{z}}} f\left(d_{j}\right)}
$$

Four different weightings were compared to the single threshold method. The four weightings are termed, equal, $\sigma$, variance, and $P_{E}$-weighting. $P_{E}$-weighting uses the conditional risk of incorrect fix for $\beta=1$.

TABLE I

Weighting Function Alternatives Considered

\begin{tabular}{ccccc}
\hline Weighting & Equal & $\sigma$ & $\sigma^{2}$ & $P_{E}$ \\
\hline$f\left(d_{i}\right)$ & 1 & $\sqrt{d_{i}}$ & $d_{i}$ & $2 \Phi\left(\frac{-1 / 2}{\sqrt{d_{i}}}\right)$ \\
\hline
\end{tabular}

When tested for a variety of models with $P_{C F}$ ranging from .85 to .9999 and a wide range of $P_{F, \text { spec }}$, it was found that $P_{E}$-weighting produces the highest availability of the fixed solution for all models studied. These models included instances with flat spectra; i.e., $\max \left\{d_{i}\right\} / \min \left\{d_{i}\right\}<1.1$, and for spectra with significant discontinuities; i.e., $\max \left\{d_{i}\right\} / \min \left\{d_{i}\right\}>$ 7. Even when performing local optimization starting from the $P_{E}$-weighted aperture, there has not been observed more than a $0.03 \%$ improvement in availability. It is therefore recommended that the aperture be sized using $P_{E}$-weighting.
This approach can yield several percent improvement in the availability of full ambiguity resolution over using a single threshold value.

\section{VAlidation of MAP GiAB Via Monte CARlo SIMULATION}

To validate the various facets of GIAB, Monte Carlo simulations were performed on multiple float solution models. For each model, the simulation was initialized by performing the decorrelating Z-transform and using $P_{E}$-weighting to set the integer aperture. Then a large sample was drawn from the distribution to generate the float solution errors, including the float baseline and float ambiguities. Next, the GIAB algorithm is applied to each float solution. Finally, the test statistics were logged, including the number of correct fixes for each value of $n_{\text {fix }}$, the number of incorrect fixes tabulated by the first errant ambiguity, and the fixed baseline error.

The sample size has been chosen to ensure that a statistically significant number of failures occurs or a significant number of solutions is available for each value of $n_{\mathrm{fix}}$. The theoretical predictions of this paper are then compared to the simulated results.To examine the goodness of fit between theory and simulation, the differences between predicted and simulated probabilities are normalized by the expected standard deviation in the measured rate. Once the probabilities $P_{S, i}$ and $P_{F, i}$ are verified, a histogram of the baseline error is compared to the a priori distributions predicted by this paper.

Several models were simulated to illustrate a range of failure rates and fixing probabilities. Small models with only 7 ambiguities are presented in full detail to save space, but similar results were obtained for $n_{\mathrm{z}} \in\{14,21,28\}$. In the following tables, $\mathcal{E}$ is one of the success or failure events, $P_{\mathcal{E}}$ are the predicted event probabilities, $\hat{P}_{\mathcal{E}}$ are the event probabilities as estimated from the Monte Carlo simulation, and $k \sigma_{P_{\mathcal{E}}}$ is the number of standard deviations difference between the predicted and estimated event probabilities. The standard deviation is based on the Beta distribution, which is the conjugate prior for the probability parameter of a binomial distribution.

Table II shows the simulation results for $n_{M C}=10^{6}$ Monte Carlo samples from a float distribution with a bootstrap probability of correct fix $P_{C F, B}=0.988$ for $P_{F, \text { spec }}=10^{-5}$. This weak model was chosen to validate the event probabilities when partial fixing must be employed frequently. The final column indicates the number of standard deviations difference 


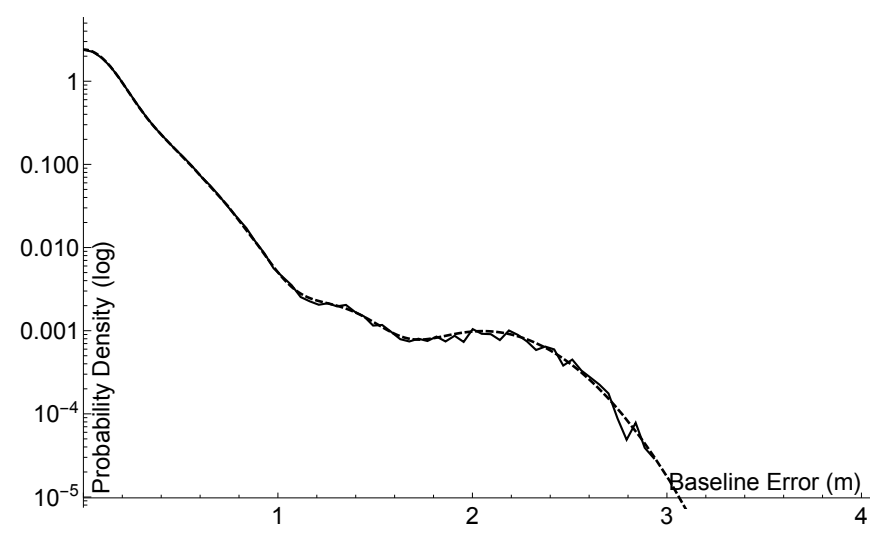

Fig. 6. The theoretical prior distributions of MAP GIAB (dashed line) and simulation histogram of the same (solid line), plotted in log scale for $n_{\mathrm{z}}=7, P_{F}=10^{-5}$, and a sample size of $2 \times 10^{6}$. The underlying model has a bootstrap probability of correct fix $P_{C F, B}=.988$. The distributions are symmetric about zero, so only the positive portion is shown. There is strong agreement between the predicted and simulated distributions. There was similar agreement for each of the intermediate distributions.

between the theoretical and simulated probabilities. The prior distribution for the model is shown with its empirical approximation in Fig. 6.

TABLE II

Predicted vs Simulated Event Probabilities for a Weak Model

\begin{tabular}{cccc}
\hline $\mathcal{E}$ & $P_{\mathcal{E}}$ & $\hat{P}_{\mathcal{E}}$ & $k \sigma_{P_{\mathcal{E}}}$ \\
\hline$F$ & 0.00001 & 0.000012 & -0.57735 \\
$S_{0}$ & 0.13872 & 0.138154 & 1.62546 \\
$S_{1}$ & 0.09308 & 0.092961 & 0.41219 \\
$S_{2}$ & 0.08423 & 0.084818 & -2.09811 \\
$S_{3}$ & 0.09987 & 0.100039 & -0.57589 \\
$S_{4}$ & 0.07162 & 0.071419 & 0.76184 \\
$S_{5}$ & 0.08010 & 0.080193 & -0.35461 \\
$S_{6}$ & 0.03362 & 0.033531 & 0.47671 \\
$S_{7}$ & 0.39878 & 0.398873 & -0.19793 \\
\hline
\end{tabular}

Table III shows the simulation results for $n_{M C}=2.2 \times 10^{8}$ Monte Carlo samples from a float distribution with a bootstrap probability of correct fix $P_{C F, B}=1-2 \times 10^{-5}$ for $P_{F, \text { spec }}=10^{-8}$. This strong model was chosen to validate the event probabilities when partial fixing is rarely relied upon. The prior distribution for the model is shown with its empirical approximation in Fig. 7. The predictions of both the weak and strong models match the simulation results well.

TABLE III

Predicted vs Simulated Event Probabilities For a Strong MODEL

\begin{tabular}{lccc}
\hline $\mathcal{E}$ & $P_{S, n}$ & $\frac{\#\left\{S_{n}\right\}}{n_{M C}}$ & $k \sigma_{P_{\mathcal{E}}}$ \\
\hline$F$ & $10^{-8}$ & $9.09 \mathrm{e}-9$ & 0.13484 \\
$S_{0}$ & 0.0007217 & 0.000720 & 0.72972 \\
$S_{1}$ & 0.0004399 & 0.000443 & -1.9805 \\
$S_{2}$ & 0.0005133 & 0.000515 & -0.9962 \\
$S_{3}$ & 0.0011997 & 0.001100 & -0.1132 \\
$S_{4}$ & 0.0009000 & 0.000901 & -0.5220 \\
$S_{5}$ & 0.0018919 & 0.001898 & -2.0746 \\
$S_{6}$ & 0.0003955 & 0.000394 & 1.13087 \\
$S_{7}$ & 0.9939380 & 0.993929 & -1.7059 \\
\hline
\end{tabular}

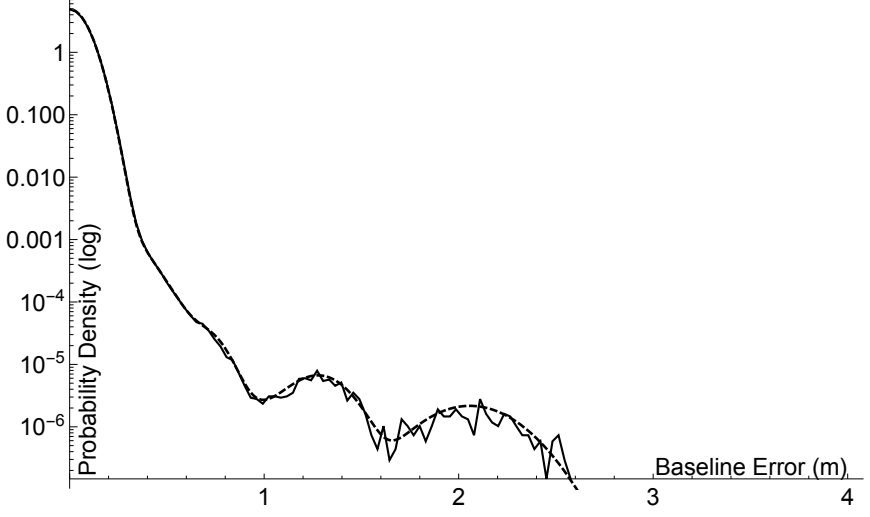

Fig. 7. The theoretical prior distributions of MAP GIAB (dashed line) and simulation histogram of the same (solid line), plotted in log scale for $n_{\mathrm{z}}=7$ a bootstrap probability of correct fix $P_{F}=10^{-8}$, and a sample size of $2.2 \times 10^{8}$.The underlying model has a bootstrap probability of correct fix $P_{C F, B}=1-2 \times 10^{-5}$. The distributions are symmetric about zero, so only the positive portion is shown. There is strong agreement between the predicted and simulated distributions. Note that since this plot is in log scale, very few samples difference appear to have a much larger impact at low probabilities.

\section{PERFormance ANALYsis}

\section{A. Protection Levels}

Integrity requirements are specified in terms of an integrity risk, $I R_{\mathrm{spec}}$, that the baseline estimation error will exceed the $A L$ threshold without warning. $I R_{\text {spec }}$ is derived from an overall risk requirement, such as probability of loss of aircraft, and is typically a fixed value for a given system use case. The $A L$ is related to physical obstacle clearance requirements, which are constant for a particular land based runway and a given aircraft. However, obstacle clearance margins are not constant when landing on a moving platform, such as an aircraft carrier at sea. Since the risk of excessive error might be evaluated against a time-varying threshold for a fixed allowable risk, it is useful to determine a protection level $(P L)$ that bounds the estimation error to the required level of risk.

A $P L$ can be thought of as the minimum $A L$ that could be met by a navigation system or algorithm for a given value of $I R_{\text {spec }}$. In terms of statisical hypothesis testing, $I R_{\text {spec }}$ corresponds to the desired confidence level, the $A L$ corresponds to the decision threshold, and the $P L$ to a prediction interval. If the risk of excess error is given as a function of $A L$, then the $P L$ can be defined as

$$
P L \triangleq \min \left\{A L \mid R(A L) \leq I R_{\text {spec }}\right\}
$$

A $P L$ for EPIC or for any version of GIAB can be computed by using a root solving method to solve (56) with $R(A L)$ defined by (25) or (30), respectively.

\section{B. Comparison to EPIC}

To demonstrate the performance of GIAB compared to the state-of-the-art high-integrity algorithm, the performance of EPIC and MAP GIAB will be compared for the measurement models previously examined. MAP GIAB is chosen because it provides better accuracy than float GIAB and is simpler to implement than MMSE GIAB. If MMSE GIAB were 
used, it would compare even more favorably with EPIC since MMSE GIAB always produces smaller $P L$ s than MAP GIAB. Because the EPIC algorithm uses an a priori, model driven approach to validation, EPIC will always produce the same $P L$ for the same number of integers fixed with a given measurement model and $I R$ requirement. Conversely, GIAB is a data-driven algorithm for which the $P L$ is a random variable. The $P L$ has a finite support since it is driven by $\left|\breve{\epsilon}_{\mathrm{c}, i}\right|<\frac{1}{2}$. For this reason, the $P L$ produced by EPIC will be compared to the minimum, maximum, and average $P L$ produced by GIAB for each number of integers fixed, along with the probability that GIAB will fix that number of integers for each model considered. An example of the a priori probability density functions of the EPIC and GIAB baseline errors is given in figure 8 .

As can be seen in table IV and table V, GIAB is able to provide smaller $P L$ s than EPIC most of the time. All distance units in these tables are in meters. Note that the $P L$ computed for $S_{0}$ by EPIC is simply the PL of the float solution with no incorrect fixing bias. The event $S_{0}$ for GIAB corresponds to the case where the measurements are so poor that no integers can be fixed successfully. The worst case $P L$ computed for any $n_{\mathrm{fix}}>0$ by MAP GIAB, which has the largest $P L \mathrm{~s}$ of any of the GIAB implementations, is better than the best $P L$ computed by EPIC.

GIAB provides lower $P L \mathrm{~s}$ because it is able to reject and exclude most of the incorrect fixes that EPIC must protect against. This implies that GIAB will also provide superior availability of integrity for models similar to those examined in this paper. It is expected that this will be the case in general since the a posteriori alternate candidate fix used in GIAB will virtually always be among the candidates considered a priori by EPIC. This implies that any decrease in the $P L$ computed by EPIC as compared to GIAB will result only when the incorrect fixing bias of the GIAB alternative fix is the same as the largest incorrect fixing bias considered by EPIC, which will be a rare event. This is why GIAB is expected to provide better availability of integrity than EPIC under most circumstances.

TABLE IV

COMPARISON OF EPIC AND GIAB FOR A WEAK MODEL

\begin{tabular}{ccccccc}
\hline $\mathcal{E}$ & $P_{\mathcal{E}}$ & $\sigma(m)$ & $P L_{\min }$ & $\mathbb{E}[P L]$ & $P L_{\max }$ & $P L_{E P I C}$ \\
\hline$S_{0}$ & 0.138 & 0.465 & 2.60 & 2.93 & 3.57 & 2.03 \\
$S_{1}$ & 0.093 & 0.332 & 1.54 & 1.56 & 1.59 & 2.86 \\
$S_{2}$ & 0.084 & 0.294 & 1.51 & 1.78 & 2.33 & 2.86 \\
$S_{3}$ & 0.099 & 0.207 & 1.68 & 1.87 & 2.24 & 2.91 \\
$S_{4}$ & 0.071 & 0.173 & 1.05 & 1.20 & 1.50 & 3.26 \\
$S_{5}$ & 0.080 & 0.126 & 0.97 & 1.08 & 1.29 & 3.14 \\
$S_{6}$ & 0.033 & 0.121 & 0.57 & 0.60 & 0.77 & 3.31 \\
$S_{7}$ & 0.398 & 0.121 & 0.54 & 0.54 & 0.54 & 3.33 \\
\hline
\end{tabular}

Note that the PLs computed by GIAB and EPIC do not increase or decrease uniformly. For example, the maximum PL increases from $1.37 \mathrm{~m}$ to $2.13 \mathrm{~m}$ for the strong model when transitioning from the first successful fix to the second successful fix. Recall that the PL is driven primarily by the bias between the most likely fix and the incorrect fixes of non-negligible probability. Because these biases depend on the relationships among the various integers and the baseline
TABLE V

COMPARISON OF EPIC AND GIAB FOR A STRONG MODEL

\begin{tabular}{ccccccc}
\hline $\mathcal{E}$ & $P_{\mathcal{E}}$ & $\sigma(m)$ & $P L_{\min }$ & $\mathbb{E}[P L]$ & $P L_{\max }$ & $P L_{E P I C}$ \\
\hline$S_{0}$ & 0.00072 & 0.310 & 2.79 & 2.92 & 3.35 & 1.77 \\
$S_{1}$ & 0.00043 & 0.221 & 1.31 & 1.32 & 1.37 & 2.62 \\
$S_{2}$ & 0.00051 & 0.196 & 1.63 & 1.74 & 2.13 & 2.61 \\
$S_{3}$ & 0.00119 & 0.138 & 1.75 & 1.83 & 2.09 & 2.49 \\
$S_{4}$ & 0.00090 & 0.115 & 1.09 & 1.16 & 1.38 & 3.03 \\
$S_{5}$ & 0.00189 & 0.084 & 0.99 & 1.04 & 1.20 & 2.91 \\
$S_{6}$ & 0.00039 & 0.081 & 0.48 & 0.53 & 0.69 & 2.99 \\
$S_{7}$ & 0.99393 & 0.081 & 0.46 & 0.46 & 0.46 & 2.95 \\
\hline
\end{tabular}

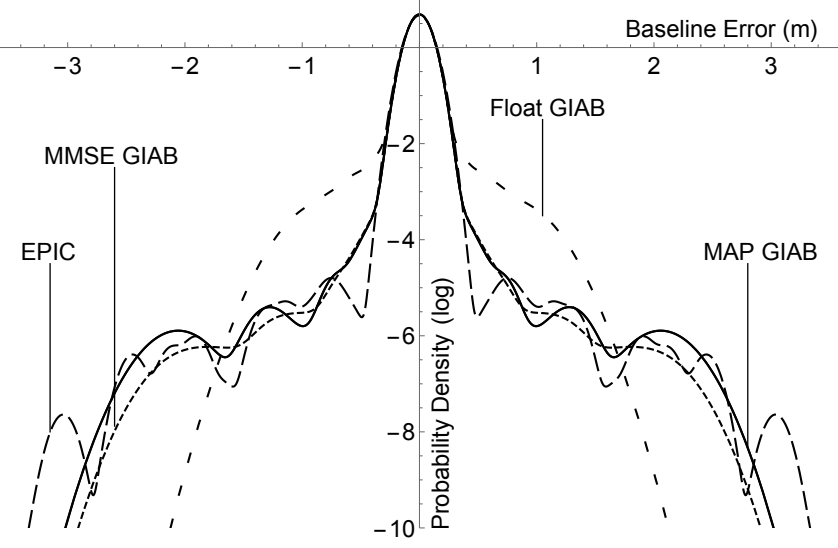

Fig. 8. The a priori distributions of EPIC and GIAB for the same model as in Fig. 7. The a priori probabilities of large errors while using GIAB are somewhat smaller than when using EPIC for this model, but GIAB provides much smaller protection levels since it uses a posteriori integrity protection.

directions of interest (e.g. vertical error), the biases can change dramatically from one integer fix to the next.

It is tempting to think that in the case of successfully fixing only two integers in the example above that it would be better to only fix one integer since that would yield a lower protection level. It may in fact be preferable to do so, but only if the bias induced in the solution by leaving the second integer floating produces acceptable accuracy performance. That is, the reduction in PL is obtained only at the expense of a biased solution which hurts average accuracy. The impact to average accuracy can be seen in the strength of the central modes of float and MAP GIAB in Fig. 8 and in the intermediate distributions of figure 4 .

\section{CONCLUSIONS}

A new data-driven CDGPS partial ambiguity resolution and validation algorithm has been developed analytically and validated with Monte Carlo simulation. The new algorithm has advantages over the state-of-the-art in that (1) datadriven methods offer improved availability of integrity over model-driven methods such as EPIC, (2) the integrity risk due to incorrect fixing is precisely controlled analytically as compared to functional approximation methods used with the ratio test and similar integer aperture methods, (3) it correctly accounts for the integrity risk of partial ambiguity resolution in the position domain that existing GIA methods neglect, and (4) it requires less computational burden than GERAFS or 
EPIC since it eliminates the search for many alternate fix candidates. The new algorithm has been shown to provide superior performance to the current state-of-the-art methods for a range of measurement models.

\section{ACKNOWLEDGMENTS}

The authors gratefully acknowledge the Naval Air Systems Command (NAVAIR) of the U.S. Navy for supporting this research. However, the opinions discussed here are those of the authors and do not necessarily represent those of the U.S. Navy or any other affiliated agencies. Mr. Green is thankful for the support of his colleagues at Coherent Technical Services,

Inc., particularly Surjan Dogra for his insightful reviews of this work.

\section{REFERENCES}

[1] Wu, S., Peck, S. R., Fries, R. M., and McGraw, G. A., "Geometry Extra-Redundant Almost Fixed Solutions: A High Integrity Approach for Carrier Phase Ambiguity Resolution for High Accuracy Relative Navigation," Position, Location and Navigation Symposium 2008 IEEE/ION, 2008, pp. 568-582.

[2] Khanafseh, S. and Pervan, B., "A new approach for calculating position domain integrity risk for cycle resolution in carrier phase navigation systems," Position, Location and Navigation Symposium, 2008 IEEE/ION, May 2008, pp. 583-591.

[3] Khanafseh, S., Joerger, M., and Pervan, B., "Integrity risk of cycle resolution in the presence of bounded faults," Position Location and Navigation Symposium (PLANS), 2012 IEEE/ION, April 2012, pp. 664 672.

[4] Teunissen, P. J. G., "The probability distribution of the ambiguity bootstrapped GNSS baseline," Journal of Geodesy, Vol. 75, No. 5-6, 2001, pp. 267-275.

[5] Teunissen, P. J. G., de Jonge, P. J., and Tiberius, C. C. J. M., "The leastsquares ambiguity decorrelation adjustment: its performance on short GPS baselines and short observation spans," Journal of Geodesy, Vol. 71, No. 10, 1997, pp. 589-602.

[6] Teunissen, P., "Integer aperture bootstrapping: a new GNSS ambiguity estimator with controllable fail-rate," Journal of Geodesy, Vol. 79, No. 6-7, 2005, pp. 389-397.

[7] Teunissen, P., "A carrier phase ambiguity estimator with easy-to-evaluate fail-rate," Artificial Satellites, Vol. 38, No. 3, 2003, pp. 89-96.

[8] Verhagen, S. and Teunissen, P., "The ratio test for future GNSS ambiguity resolution," GPS Solutions, Vol. 17, No. 4, 2013, pp. 535-548.

[9] Zhang, J., Wu, M., Li, T., and Zhang, K., "Integer aperture ambiguity resolution based on difference test," Journal of Geodesy, Vol. 89, No. 7, 2015, pp. 667-683

[10] Verhagen, S. and Teunissen, P., "New Global Navigation Satellite System Ambiguity Resolution Method Compared to Existing Approaches," Journal of Guidance, Control, and Dynamics, Vol. 29, No. 4, 2006, pp. 981-991.

[11] Teunissen, P. and Verhagen, S., "The GNSS ambiguity ratio-test revisited: a better way of using it," Survey Review, Vol. 41, No. 312, 2009, pp. $138-151$.

[12] Wang, L. and Verhagen, S., "A new ambiguity acceptance test threshold determination method with controllable failure rate," Journal of Geodesy, Vol. 89, No. 4, 2015, pp. 361-375.

[13] Brack, A., "On reliable data-driven partial GNSS ambiguity resolution," GPS Solutions, Vol. 19, No. 3, 2015, pp. 411-422.

[14] Teunissen, P. J. G., "GNSS ambiguity resolution with optimally controlled failure-rate," Artificial Satellites, Vol. 40, 2005, pp. 219-227.

[15] Brack, A. and Gnther, C., "Generalized integer aperture estimation for partial GNSS ambiguity fixing," Journal of Geodesy, Vol. 88, No. 5, 2014, pp. 479-490.

[16] Teunissen, P., "Theory of carrier phase ambiguity resolution," Wuhan University Journal of Natural Sciences, Vol. 8, No. 2, 2003, pp. 471484.

NAVAIR Public Release SPR 2016-54

Distribution Statement A- Approved for public release; distribution is unlimited. 